\title{
A NEW PERTURBATION BOUND FOR THE LDU FACTORIZATION OF DIAGONALLY DOMINANT MATRICES*
}

\author{
MEGAN DAILEY ${ }^{\dagger}$, FROILÁN M. DOPICO ${ }^{\ddagger}$, AND QIANG YE ME $^{\S}$
}

\begin{abstract}
This work introduces a new perturbation bound for the $L$ factor of the LDU factorization of (row) diagonally dominant matrices computed via the column diagonal dominance pivoting strategy. This strategy yields $L$ and $U$ factors which are always well-conditioned and, so, the LDU factorization is guaranteed to be a rank-revealing decomposition. The new bound together with those for the $D$ and $U$ factors in [F. M. Dopico and P. Koev, Numer. Math., 119 (2011), pp. 337371 ] establish that if diagonally dominant matrices are parameterized via their diagonally dominant parts and off-diagonal entries, then tiny relative componentwise perturbations of these parameters produce tiny relative normwise variations of $L$ and $U$ and tiny relative entrywise variations of $D$ when column diagonal dominance pivoting is used. These results will allow us to prove in a follow-up work that such perturbations also lead to strong perturbation bounds for many other problems involving diagonally dominant matrices.
\end{abstract}

Key words. accurate computations, column diagonal dominance pivoting, diagonally dominant matrices, diagonally dominant parts, LDU factorization, rank-revealing decomposition, relative perturbation theory

AMS subject classifications. 65F05, 65F15, 15A18, 15A23, 15B99

DOI. $10.1137 / 13093858 \mathrm{X}$

1. Introduction. Perturbation analysis is a classical topic in matrix theory and numerical linear algebra [23, 24, 35] which still attracts a lot of attention. In recent years, considerable effort has been devoted to deriving sharper perturbation bounds when structured perturbations of important classes of structured matrices are considered (see, as a sample, $[1,3,4,7,12,14,18,21,22,26,27,28,29,30,31,33,34,37$, $38,40])$. In this paper, we present a new perturbation bound for the $L$ factor of the LDU factorization of diagonally dominant matrices under a class of componentwise structure-preserving perturbations which are important in numerical computations $[14,39,40]$. Here, $A=L D U$ is an LDU factorization of $A$ if $L$ is a unit lower triangular matrix, $D$ is a diagonal matrix, and $U$ is a unit upper triangular matrix.

Solution of this problem is motivated by several facts. First, apart from its classical applications [20], the LDU factorization has been applied recently to computing accurate rank-revealing decompositions (RRD) [11] of many classes of structure matrices, which are used to perform matrix computations with high relative accuracy $[5,11,13,15,17]$. In this context, an LDU factorization is an RRD if $L$ and $U$ are well-conditioned. A key point in computing an LDU factorization as an RRD is that the standard partial pivoting strategy does not produce, in general, well-conditioned factors $L$ and $U$, and that neither complete nor rook pivoting guarantees that $L$ and

* Received by the editors September 25, 2013; accepted for publication (in revised form) by J. L. Barlow May 7, 2014; published electronically July 8, 2014.

http://www.siam.org/journals/simax/35-3/93858.html

$\dagger$ Indiana University Kokomo, Kokomo, IN 46904-9003 (medailey@iuk.edu). This author was supported in part by NSF under grant DMS- 1318633.

${ }^{\ddagger}$ Instituto de Ciencias Matemáticas CSIC-UAM-UC3M-UCM and Departamento de Matemáticas, Universidad Carlos III de Madrid, 28911 Leganés, Spain (dopico@math.uc3m.es). This author was supported in part by Ministerio de Economía y Competitividad of Spain under grant MTM201232542.

$\S$ Department of Mathematics, University of Kentucky, Lexington, KY 40506 (qiang.ye@uky.edu). This author was supported in part by NSF under grant DMS-1318633.

904 
$U$ are well-conditioned, although they often do so in practice. Therefore, it may be necessary to consider other special pivoting strategies that lead to LDU factorizations that are guaranteed to be RRDs.

Another motivation for this work comes from the fact that the LDU factorization may not be accurately computed in a forward stable manner in general. This follows from the perturbation theory of the LDU factorization, since the sharpest perturbation bounds available in the literature $[8,9]$ show that large changes in the factors may happen under tiny perturbations of the matrix in certain cases. In addition, although complete and rook pivoting improve the sensitivity of the LDU factorization $[6,8]$, extremely large variations of the factors are still possible. Therefore, backward stable algorithms for the LDU factorization do not necessarily lead to accurate factors.

However, highly accurate matrix algorithms based on RRDs [5, 11, 13, 15, 17] require an LDU factorization computed accurately in the following sense: $L$ and $U$ are computed with tiny normwise relative errors and $D$ is computed with tiny entrywise relative errors. These strict requirements imply that accurate LDU factorizations can be computed only for some special structured matrices via algorithms carefully designed to exploit the corresponding structures (see $[11,15,17])$.

Diagonally dominant matrices arise in many applications and have many favorable properties [20, 24]. For brevity, we focus on row diagonally dominant matrices, although the results we present hold for the column case by taking transposes. For these matrices, the LDU factorization without pivoting satisfies that $U$ is also row diagonally dominant and, so, well-conditioned with $\kappa_{\infty}(U) \leq 2 n$ [32]. In contrast, $L$ may not be well-conditioned, but if the factorization is computed with a pivoting strategy introduced in $[32,39]$, then $L$ is column diagonally dominant and, so, well-conditioned with $\kappa_{1}(L) \leq 2 n$ [32]. This strategy is called here column diagonal dominance pivoting and is specific for row diagonally dominant matrices. It guarantees that the LDU factorizations are RRDs.

An accurate algorithm for computing the LDU factorization of row diagonally dominant matrices has been developed recently in [39]. It works for any pivoting strategy that preserves the diagonally dominant structure and, in particular, for column diagonal dominance pivoting. The accuracy follows from an error analysis based on the perturbation theory for the LDU factorization of row diagonally dominant matrices presented in [14]. However, the perturbation bound on $L$ in [14] is guaranteed to be small only if complete pivoting is used. Unfortunately, this pivoting strategy does not guarantee that $L$ is well-conditioned. Then, a fundamental question is whether or not a strong perturbation bound for $L$ still holds for the LDU factorization produced by the column diagonal dominance pivoting.

This paper extends the results in [14] to prove a new perturbation bound for $L$ under the assumption that column diagonal dominance pivoting is used. This together with the results in [14] demonstrates that a diagonally dominant matrix has an LDU factorization that is an RRD and is stable under perturbation. Among other applications, this bound is crucial in a separate work [10] that studies perturbation properties of diagonally dominant matrices for many other linear algebra problems.

The strong perturbation results proved here and in [14] are based on parameterizing row diagonally dominant matrices by its off-diagonal entries and diagonally dominant parts and on considering small relative componenwise perturbations of these parameters. This parametrization was introduced in [39], used in [40] to derive relative perturbation bounds for eigenvalues of positive semidefinite matrices, and recently has led to strong perturbation bounds for many other problems [10].

Copyright () by SIAM. Unauthorized reproduction of this article is prohibited. 
The results in this paper and in [14] show that column diagonal dominance pivoting has two properties that make it optimal for row diagonally dominant matrices: it computes an LDU factorization that is stable under perturbations of off-diagonal entries and diagonally dominant parts and whose $L$ and $U$ factors are always wellconditioned. As far as we know, no other pivoting strategy enjoys these two favorable properties.

The rest of this paper is organized as follows. In section 2, we give an overview of diagonally dominant matrices and revise previous results that are needed in this work. Section 3 presents the new perturbation bound for the $L$ factor and its proof. Finally, in section 4, conclusions and lines of future research are discussed. Before proceeding, we present below the notation used in this paper.

Notation. In this paper we consider only real matrices and we denote by $\mathbb{R}^{m \times n}$ the set of $m \times n$ real matrices. The entries of a matrix $A$ are $a_{i j}$ and $|A|$ is the matrix with entries $\left|a_{i j}\right|$. The inequality $A \geq B$ for matrices means $a_{i j} \geq b_{i j}$ for all $i, j$, and the inequality $v \geq w$ for vectors means $v_{i} \geq w_{i}$ for all $i$. Analogously, the inequality $v \geq 0$ for the vector $v$ means $v_{i} \geq 0$ for all $i$. We use the MATLAB notation for submatrices. That is, $A(i: j, k: l)$ denotes the submatrix of $A$ formed by rows $i$ through $j$ and columns $k$ through $l$. We use $A\left(i^{\prime}, j^{\prime}\right)$ to denote the submatrix of $A$ formed by deleting row $i$ and column $j$ from $A$. Let $\alpha=\left[i_{1}, i_{2}, \ldots, i_{p}\right]$, where $1 \leq i_{1}<i_{2}<\cdots<i_{p} \leq m$, and $\beta=\left[j_{1}, j_{2}, \ldots, j_{q}\right]$, where $1 \leq j_{1}<j_{2}<\cdots<j_{q} \leq n$. Then $A(\alpha, \beta)$ denotes the submatrix of $A$ that consists of rows $i_{1}, i_{2}, \ldots, i_{p}$ and columns $j_{1}, j_{2}, \ldots, j_{q}$. In MATLAB notation, $1: k$ denotes the row vector $[1,2, \ldots, k]$. For convenience, we also use the notation $1: k$ to denote the set $\{1,2, \ldots, k\}$. We denote by $I_{s}$ the $s \times s$ identity matrix and by $0_{s}$ the $s \times s$ zero matrix. Two matrix norms will be used: $\|A\|_{1}=\max _{j} \sum_{i}\left|a_{i j}\right|$ and $\|A\|_{\infty}=\max _{i} \sum_{j}\left|a_{i j}\right|$. The condition numbers of a nonsingular matrix $A$ in any of these norms is denoted as $\kappa_{i}(A):=\|A\|_{i}\left\|A^{-1}\right\|_{i}$ for $i=1, \infty$. The sign of $x \in \mathbb{R}$ is $\operatorname{sign}(x)$, where $\operatorname{sign}(0)$ is defined to be 1 .

2. Preliminaries. In this section, we give an overview of diagonally dominant matrices and some of their properties. More information on this topic can be found in $[14$, section 2] and $[24,25]$.

Definition 2.1. A matrix $A=\left[a_{i j}\right] \in \mathbb{R}^{n \times n}$ is said to be row diagonally dominant if $\left|a_{i i}\right| \geq \sum_{j \neq i}\left|a_{i j}\right|$ for $i=1, \ldots, n$.

In the rest of the paper, all row diagonally dominant matrices $A \in \mathbb{R}^{n \times n}$ that are considered satisfy $a_{i i} \geq 0$ for $i=1, \ldots, n$. This does not impose any restriction for studying LDU factorizations, since we can multiply $A$ by a diagonal matrix $S$ with diagonal entries equal to \pm 1 to get this property, and the LDU factorizations of $A$ and $S A$ are trivially related each other.

An idea that has played a key role in deriving relative perturbation bounds and high relative accuracy algorithms for row diagonally dominant matrices [39, 40, 14] is to reparameterize these matrices in terms of their diagonally dominant parts and off-diagonal entries as follows.

DEFINITION 2.2.

(1) Given a matrix $M=\left[m_{i j}\right] \in \mathbb{R}^{n \times n}$ and a vector $v=\left[v_{i}\right] \in \mathbb{R}^{n}$, we use $\mathcal{D}(M, v)$ to denote the matrix $A=\left[a_{i j}\right] \in \mathbb{R}^{n \times n}$ whose off-diagonal entries are the same as $M$ (i.e., $a_{i j}=m_{i j}$ for $i \neq j$ ) and whose ith diagonal entry is $a_{i i}=v_{i}+\sum_{j \neq i}\left|m_{i j}\right|$ for $i=1, \ldots, n$.

(2) Given a matrix $A=\left[a_{i j}\right] \in \mathbb{R}^{n \times n}$, we denote by $A_{D} \in \mathbb{R}^{n \times n}$ the matrix whose off-diagonal entries are the same as $A$ and whose diagonal entries are zero. 
Then, letting $v_{i}=a_{i i}-\sum_{j \neq i}\left|a_{i j}\right|$ for $i=1, \ldots, n$, and $v=\left[v_{1}, v_{2}, \ldots, v_{n}\right]^{T} \in$ $\mathbb{R}^{n}$, we have

$$
A=\mathcal{D}\left(A_{D}, v\right)
$$

and we call it the representation of $A$ by its diagonally dominant parts $v$ and off-diagonal entries $A_{D}$.

Observe that in the representation $A=\mathcal{D}\left(A_{D}, v\right) \in \mathbb{R}^{n \times n}$ the condition $v \geq 0$ is equivalent to the statement that $A$ is row diagonally dominant and has nonnegative diagonal entries. We emphasize that the condition $v \geq 0$ will be used as an assumption in most of the results in section 3 and, so, the reader should bear in mind its underlying meaning.

Theorem 2.3 lists some basic properties of diagonally dominant matrices that are often used in this work; see, e.g., [14, Theorem 1].

TheOREM 2.3. If $A \in \mathbb{R}^{n \times n}$ is row diagonally dominant, then

(a) every principal submatrix of $A$ is row diagonally dominant;

(b) $P A P^{T}$ is row diagonally dominant for any permutation matrix $P \in \mathbb{R}^{n \times n}$;

(c) if $a_{11} \neq 0$ then the Schur complement of $a_{11}$ in $A$ is row diagonally dominant;

(d) if $\operatorname{det} A \neq 0$ then $\operatorname{det} A$ has the same sign as the product $a_{11} a_{22} \cdots a_{n n}$; and

(e) $\left|\operatorname{det} A\left(i^{\prime}, i^{\prime}\right)\right| \geq\left|\operatorname{det} A\left(i^{\prime}, j^{\prime}\right)\right|$ for all $i=1, \ldots, n$ and for all $j \neq i$, where we recall that $A\left(i^{\prime}, j^{\prime}\right)$ denotes the submatrix of $A$ formed by deleting row $i$ and column $j$ of $A$.

Diagonally dominant matrices have several other nice properties that are useful. For instance, strictly row diagonally dominant matrices (i.e., row diagonally dominant matrices as defined in Definition 2.1 with strict inequalities) are nonsingular and Gaussian elimination can be performed on them without interchanging rows or columns. This implies that any strictly row diagonally dominant matrix $A$ has a unique LDU factorization. A general row diagonally dominant matrix $A$ may be rank deficient, and in this case $A$ may not have an LDU factorization. However, applying any diagonal pivoting strategy (i.e., pivoting with simultaneous and equal row and column permutations) to $A$ always leads to a matrix $P A P^{T}$ that has LDU factorization, $P A P^{T}=L D U$, where $P$ is the permutation matrix defined by the pivoting strategy. If $A$ is rank deficient, then $D$ has diagonal entries that are zero and $L$ and $U$ may not be unique, even when $P$ is fixed. We then consider the following unique form of the LDU factorization.

Definition 2.4 (see [14, Definition 1]). A row diagonally dominant matrix $A \in \mathbb{R}^{n \times n}$ with rank $r$ is said to have LDU factorization if there exist a unit lower triangular matrix $L_{11} \in \mathbb{R}^{r \times r}$, a unit upper triangular matrix $U_{11} \in \mathbb{R}^{r \times r}$, and a nonsingular diagonal matrix $D_{11} \in \mathbb{R}^{r \times r}$ such that $A=L D U$, where

$$
L=\left[\begin{array}{cc}
L_{11} & 0 \\
L_{21} & I_{n-r}
\end{array}\right], \quad D=\left[\begin{array}{cc}
D_{11} & 0 \\
0 & 0_{n-r}
\end{array}\right], \quad U=\left[\begin{array}{cc}
U_{11} & U_{12} \\
0 & I_{n-r}
\end{array}\right] .
$$

Throughout the paper, the LDU factorization refers to the one as defined above. The nontrivial entries of the $L, D$, and $U$ factors in Definition 2.4 can be expressed in terms of minors of $A$. This is a classical result that we recall in Theorem 2.5, since it is fundamental in section 3 , where the new perturbation bounds for the LDU factorization are obtained via detailed perturbation properties of the minors of $A$.

Theorem 2.5 (see $\left[19\right.$, p. 35]). If $A \in \mathbb{R}^{n \times n}$ has rank $r$ and has LDU factorization as in Definition 2.4, then this factorization is unique and the nontrivial entries 
of $L=\left[l_{i j}\right], D=\operatorname{diag}\left[d_{1}, \ldots, d_{r}, 0, \ldots, 0\right]$, and $U=\left[u_{i j}\right]$ are given by

$$
\begin{aligned}
l_{i j} & =\frac{\operatorname{det} A([1: j-1, i], 1: j)}{\operatorname{det} A(1: j, 1: j)}, \quad i>j \text { and } j=1, \ldots, r, \\
d_{i} & =\frac{\operatorname{det} A(1: i, 1: i)}{\operatorname{det} A(1: i-1,1: i-1)}, \quad i=1, \ldots, r, \\
u_{i j} & =\frac{\operatorname{det} A(1: i,[1: i-1, j])}{\operatorname{det} A(1: i, 1: i)}, \quad i<j \text { and } i=1, \ldots, r,
\end{aligned}
$$

where we define $\operatorname{det} A(1: 0,1: 0):=1$.

Next, we revise two particular diagonal pivoting strategies which are used to compute via Gaussian elimination LDU factorizations of a row diagonally dominant matrix $A$ that are RRDs, i.e., with both factors $L$ and $U$ well-conditioned. First, recall that any diagonal pivoting strategy applied on $A$ leads to a factor $U$ that is also row diagonally dominant and, therefore, well-conditioned [32]. At each stage of Gaussian elimination with complete diagonal pivoting, the same row and column are exchanged to place in the pivot position the diagonal entry with the largest absolute value of the corresponding Schur complement. Observe that for row diagonally dominant matrices, complete diagonal pivoting coincides with standard complete pivoting but, despite this fact, it does not lead to an LDU factorization that is guaranteed to be an $\mathrm{RRD}$, although in practice, the computed factor $L$ is almost always well-conditioned. The column diagonal dominance pivoting strategy mentioned in section 1 is much less known than complete diagonal pivoting, but applied on $A$ computes a factor $L$ which is column diagonally dominant and, hence, is always well-conditioned [32]. Some additional notation is needed to introduce such pivoting strategy.

In general, consider applying Gaussian elimination with a diagonal pivoting strategy to a row diagonally dominant matrix $A \in \mathbb{R}^{n \times n}$ with nonnegative diagonal entries. We assume that $A$ is arranged for that diagonal pivoting strategy, which means that the permutation defined by the pivoting is applied to $A$ in advance. In each stage Gaussian elimination makes zero all the entries below the diagonal of a certain column. Define $A^{(1)}:=A$ and define $A^{(k+1)}:=\left[a_{i j}^{(k+1)}\right] \in \mathbb{R}^{n \times n}$ to be the matrix obtained after $k$ stages of Gaussian elimination have been performed. So, all the entries below the diagonal in the first $k$ columns of $A^{(k+1)}$ are equal to zero, $A^{(k+1)}(1: k,:)=A^{(k)}(1: k,:)$, and, in addition, the following identity presented in [19] can be easily proved from the properties of determinants:

$$
a_{i j}^{(k+1)}=\frac{\operatorname{det} A([1: k, i],[1: k, j])}{\operatorname{det} A(1: k, 1: k)}
$$

for $k+1 \leq i, j \leq n$ and $1 \leq k \leq \min \{r, n-1\}$, where $r=\operatorname{rank}(A)$. It follows from Theorem $2.3(\mathrm{c})$ that $A^{(k+1)}$ is row diagonally dominant and that $A^{(k+1)}(k+1: n, k+1: n)$ is also row diagonally dominant, and from Theorems $2.3(\mathrm{a})-(\mathrm{d})$ and $(2.5)$ that $A^{(k+1)}$ has nonnegative diagonal entries. Thus, Gaussian elimination applied on $A$ generates a sequence of row diagonally dominant matrices with nonnegative diagonal entries $A^{(k)} \in \mathbb{R}^{n \times n}, k=1,2, \ldots, \min \{n, r+1\}$, such that $A^{(k)}(k: n, k: n)$ is also row diagonally dominant. Observe that this implies that there is at least one column of $A^{(k)}(k: n, k: n)$ that is diagonally dominant, i.e., $a_{i i}^{(k)}-\sum_{j=k, j \neq i}^{n}\left|a_{j i}^{(k)}\right| \geq 0$ for some $i=k, \ldots, n$. Then, the column diagonal dominance pivoting strategy arranges $A$ in 
such a way that

$$
a_{k k}^{(k)}=\max _{k \leq i \leq n}\left\{a_{i i}^{(k)}: a_{i i}^{(k)}-\sum_{j=k, j \neq i}^{n}\left|a_{j i}^{(k)}\right| \geq 0\right\},
$$

for $k=1, \ldots, r$. This pivoting strategy was suggested in [32] for matrices with other structures and used for the first time in [39] for general row diagonally dominant matrices. It is immediate to see that column diagonal dominance pivoting produces a column diagonally dominant factor $L$. Algorithmically, the column diagonal dominance pivoting is implemented by exchanging, before performing the $k$ th stage of Gaussian elimination, the same row and column to place in the pivot position $(k, k)$ the maximal diagonal entry which is column diagonally dominant to get (2.6). At the end, we obtain a row diagonally dominant factor $U$ as usual and, in addition, a column diagonally dominant factor $L$. Hence, by [32], the condition numbers of $L$ and $U$ can be bounded as

$$
\kappa_{\infty}(L) \leq n^{2}, \quad \kappa_{\infty}(U) \leq 2 n, \quad \kappa_{1}(L) \leq 2 n, \quad \kappa_{1}(U) \leq n^{2} .
$$

So, the LDU factorization of $A$ arranged with the column diagonal dominance pivoting strategy is always an RRD.

3. A new perturbation bound for the LDU factorization of diagonally dominant matrices. The perturbation bounds available in the literature for the LU and LDU factorizations of general matrices $[2,8,9,16,36]$ do not guarantee small entrywise error bounds for the $D$ factor, which is essential in computing the LDU factorization as an accurate RRD. Even for the $L$ and $U$ factors, the sharpest bounds available $[8,9]$ give only conditional stability. These results, however, appear to be the best one can get for general matrices.

For any row diagonally dominant matrix $A$, a stronger perturbation theory for its LDU factorization has been presented in [14] and has been successfully used to prove rigorously that the algorithm in [39] with complete diagonal pivoting computes an accurate LDU factorization of $A$. The key components of this theory are to use as parameters the diagonally dominant parts and the off-diagonal entries of $A$ introduced in Definition 2.2 and (2.1), and to preserve the diagonally dominant structure for getting perturbation bounds which are independent of any condition number and are always tiny for tiny perturbations. For completeness, we state as follows the main perturbation result in [14], with slightly simplified bounds.

Theorem 3.1 (see [14, Theorem 3]). Let $A=\mathcal{D}\left(A_{D}, v\right) \in \mathbb{R}^{n \times n}$ be such that $v \geq 0$. Suppose $A$ has LDU factorization $A=L D U$ (as in Definition 2.4) with $L=\left[l_{i j}\right] \in \mathbb{R}^{n \times n}, D=\operatorname{diag}\left[d_{1}, \ldots, d_{n}\right] \in \mathbb{R}^{n \times n}$, and $U=\left[u_{i j}\right] \in \mathbb{R}^{n \times n}$. Let $\tilde{A}=$ $\mathcal{D}\left(\tilde{A}_{D}, \tilde{v}\right) \in \mathbb{R}^{n \times n}$ be such that

$$
|\tilde{v}-v| \leq \epsilon v \quad \text { and } \quad\left|\tilde{A}_{D}-A_{D}\right| \leq \epsilon\left|A_{D}\right| \quad \text { for some } 0 \leq \epsilon<\frac{1}{2 n} .
$$

Then $\tilde{A}$ has LDU factorization $\tilde{A}=\tilde{L} \tilde{D} \tilde{U}$ with $\tilde{L}=\left[\tilde{l}_{i j}\right], \tilde{D}=\operatorname{diag}\left[\tilde{d}_{1}, \ldots, \tilde{d}_{n}\right]$, and $\tilde{U}=\left[\tilde{u}_{i j}\right]$ and the factors $\tilde{L}, \tilde{D}$, and $\tilde{U}$ satisfy,

(a) for $i=1, \ldots, n$,

$$
\left|\tilde{d}_{i}-d_{i}\right| \leq \frac{2 n \epsilon}{1-2 n \epsilon} d_{i}
$$

Copyright () by SIAM. Unauthorized reproduction of this article is prohibited. 
(b) for $1 \leq i<j \leq n$,

$$
\left|\tilde{u}_{i j}-u_{i j}\right| \leq 3 n \epsilon \text { and } \frac{\|\tilde{U}-U\|_{\infty}}{\|U\|_{\infty}} \leq 3 n^{2} \epsilon
$$

(c) and, if $A$ is arranged for complete diagonal pivoting, for $n \geq i>j \geq 1$,

$$
\left|\tilde{l}_{i j}-l_{i j}\right| \leq \frac{3 n \epsilon}{1-2 n \epsilon} \quad \text { and } \frac{\|\tilde{L}-L\|_{\infty}}{\|L\|_{\infty}} \leq \frac{3 n^{2} \epsilon}{1-2 n \epsilon} .
$$

Observe that the assumptions $v \geq 0$ and $0 \leq \epsilon<1 /(2 n)<1$ in Theorem 3.1 imply $\tilde{v} \geq 0$, and, so, $\tilde{A}$ is row diagonally dominant with nonnegative diagonal entries. In plain words, this means that the perturbations of $A$ considered in (3.1) preserve the diagonally dominant structure. It is interesting to remark that the original statements of parts (a) and (b) of Theorem 3.1 presented in [14] remain valid under the wider assumption $0 \leq \epsilon<1$ at the cost of somewhat more complicated statements that express $\tilde{d}_{i}$ as a multiplicative perturbation of $d_{i}$.

The perturbation bounds for $D$ and $U$ in Theorem 3.1 hold in general, but the bound on $L$ only holds if the matrix $A$ is arranged for complete diagonal pivoting. This is a critical assumption for the proof of part (c) in Theorem 3.1. Indeed, [14] provides an example where the perturbation of $L$ may be of order 1 for very small $\epsilon$ if the complete diagonal pivoting strategy is not used. As discussed in sections 1 and 2, the complete diagonal pivoting strategy, although useful for almost all matrices, does not guarantee a well-conditioned factor $L$ and, therefore, it does not compute an LDU factorization which is guaranteed to be an RRD. It is then essential to demonstrate that a rank-revealing LDU factorization such as the one produced by the column diagonal dominance pivoting strategy is stable under the structured perturbations considered in Theorem 3.1. The present paper proves precisely this, by showing that a normwise perturbation bound on $L$ similar to that in Theorem 3.1(c) holds when column diagonal dominance pivoting is used. This is the main result in this paper and is stated in Theorem 3.2.

Theorem 3.2. Let $A=\mathcal{D}\left(A_{D}, v\right) \in \mathbb{R}^{n \times n}$ be such that $v \geq 0$. Suppose that $A$ is arranged for column diagonal dominance pivoting and let $A=L D U$ be the LDU factorization (as in Definition 2.4). Let $\tilde{A}=\mathcal{D}\left(\tilde{A}_{D}, \tilde{v}\right) \in \mathbb{R}^{n \times n}$ be such that

$$
|\tilde{v}-v| \leq \epsilon v \quad \text { and } \quad\left|\tilde{A}_{D}-A_{D}\right| \leq \epsilon\left|A_{D}\right| \quad \text { for some } 0 \leq \epsilon<\frac{1}{12 n+1} .
$$

Then $\tilde{A}$ has LDU factorization $\tilde{A}=\tilde{L} \tilde{D} \tilde{U}$, parts (a) and (b) of Theorem 3.1 hold, and, in addition,

$$
\|\tilde{L}-L\|_{1} \leq \frac{2 n(4 n-1) \epsilon}{1-(12 n+1) \epsilon} .
$$

Observe that $\|L\|_{1} \geq 1$ and, as a consequence, the absolute normwise bound in (3.3) immediately implies that the same bound holds for the relative variation $\|\tilde{L}-L\|_{1} /\|L\|_{1}$. The assumption $0 \leq \epsilon<1 /(12 n+1)$ in Theorem 3.2 implies that the bound in (3.3) is well-defined.

Example 3.3. This example illustrates that column diagonal dominance pivoting is essential to guarantee, simultaneously, a good behavior of the factor $L$ under 
structured perturbations of type (3.2) and the column diagonal dominance of $L$. The entries of all $L, D$, and $U$ factors displayed in this example are the exact values of the corresponding factors. Consider, first, the LDU factorization of the following row diagonally dominant matrix $A$ :

$$
\begin{aligned}
A & =\left[\begin{array}{cccc}
1000 & 100 & 500 & 100 \\
0 & 0.1 & 0.05 & 0 \\
100 & 10 & 120 & 0 \\
0 & 0 & 69.96 & 69.97
\end{array}\right] \\
& =\left[\begin{array}{ccccc}
1 & & & \\
0 & 1 & & \\
0.1 & 0 & 1 & \\
0 & 0 & \frac{69.96}{70} & 1
\end{array}\right]\left[\begin{array}{cccc}
1000 & & \\
& 0.1 & & \\
& & & \\
& & & \\
& & \frac{55975}{700}
\end{array}\right]\left[\begin{array}{cccc}
1 & 0.1 & 0.5 & 0.1 \\
& 1 & 0.5 & 0 \\
& & 1 & \frac{-1}{7} \\
& & & 1
\end{array}\right]
\end{aligned}
$$

and note that the vector of diagonally dominant parts of $A$ is $v(A)=[300,0.05,10,0.01]$. Now consider the LDU factorization of the row diagonally dominant matrix $\tilde{A}=\tilde{L} \tilde{D} \tilde{U}$ :

$$
\begin{aligned}
& \tilde{A}=\left[\begin{array}{cccc}
1000 & 101 & 500 & 100 \\
0 & 0.1 & 0.05 & 0 \\
100 & 10 & 120 & 0 \\
0 & 0 & 69.96 & 69.97
\end{array}\right]
\end{aligned}
$$

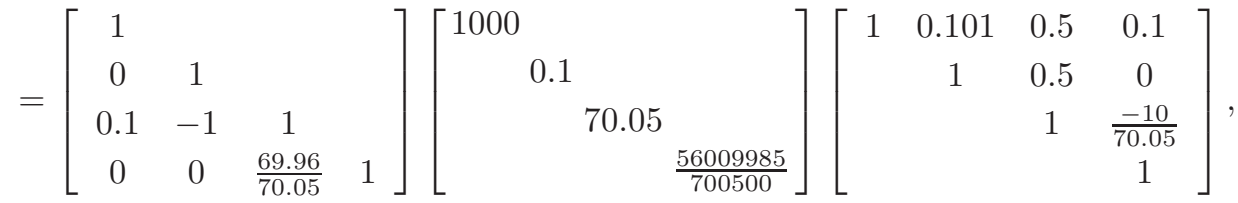

whose diagonally dominant parts are $v(\tilde{A})=[299,0.05,10,0.01]$. Note that $A$ and $\tilde{A}$ satisfy (3.2) with $\epsilon=10^{-2}$, but that their $L$ factors are very different since $\left|\tilde{l}_{32}-l_{32}\right|=$ 1. In contrast, the componentwise relative difference of the $D$ factors is smaller than $10^{-2}$, and the normwise difference of the $U$ factors is also smaller than $10^{-2}$ in the $\infty$-norm, which is in accordance with parts (a) and (b) of Theorem 3.1.

Suppose $P$ is the permutation matrix such that $P A P^{T}$ is arranged for complete diagonal pivoting; then we have

$$
\begin{aligned}
P A P^{T} & =\left[\begin{array}{cccc}
1000 & 500 & 100 & 100 \\
100 & 120 & 0 & 10 \\
0 & 69.96 & 69.97 & 0 \\
0 & 0.05 & 0 & 0.1
\end{array}\right] \\
& =\left[\begin{array}{cccccc}
1 & & & \\
0.1 & 1 & & \\
0 & \frac{69.96}{70} & 1 & \\
0 & \frac{0.05}{70} & \frac{1}{11195} & 1
\end{array}\right]\left[\begin{array}{cccc}
1000 & & & \\
& 70 & & \\
& & \frac{55975}{700} & \\
& & & 0.1
\end{array}\right]\left[\begin{array}{cccc}
1 & 0.5 & 0.1 & 0.1 \\
& 1 & \frac{-1}{7} & 0 \\
& & 1 & 0 \\
& & & 1
\end{array}\right]
\end{aligned}
$$

Copyright $\odot$ by SIAM. Unauthorized reproduction of this article is prohibited. 
and

$$
\begin{aligned}
P \tilde{A} P^{T} & =\left[\begin{array}{cccc}
1000 & 500 & 100 & 101 \\
100 & 120 & 0 & 10 \\
0 & 69.96 & 69.97 & 0 \\
0 & 0.05 & 0 & 0.1
\end{array}\right] \\
= & {\left[\begin{array}{cccc}
1 & & & \\
0.1 & 1 & & \\
0 & \frac{69.96}{70} & 1 & \\
0 & \frac{0.05}{70} & \frac{1}{11195} & 1
\end{array}\right]\left[\begin{array}{ccc}
1000 & & \\
& 70 & \\
& \frac{55975}{700} & \\
& & \frac{1120.1997}{1195}
\end{array}\right]\left[\begin{array}{cccc}
1 & 0.5 & 0.1 & 0.101 \\
& 1 & \frac{-1}{7} & \frac{-1}{700} \\
& & 1 & \frac{69.96}{55975} \\
& & & 1
\end{array}\right], }
\end{aligned}
$$

where $\frac{1120.1997}{11195} \approx 0.10006$. Observe that the $L$ factors of $P A P^{T}$ and $P \tilde{A} P^{T}$ are identical, the componentwise relative difference of the $D$ factors is smaller than $10^{-2}$, and the normwise difference of the $U$ factors is also smaller than $10^{-2}$ in the $\infty$-norm. This is in accordance with Theorem 3.1. However, observe that the $L$ factor of $P A P^{T}$ is not column diagonally dominant.

We can obtain a column diagonally dominant factor $L$ by using column diagonal dominance pivoting instead. For instance, suppose $P_{1}$ is the permutation matrix such that $P_{1} A P_{1}^{T}$ is arranged for column diagonal dominance pivoting. Then,

$$
\begin{aligned}
P_{1} A P_{1}^{T} & =\left[\begin{array}{cccc}
1000 & 100 & 500 & 100 \\
0 & 69.97 & 69.96 & 0 \\
100 & 0 & 120 & 10 \\
0 & 0 & 0.05 & 0.1
\end{array}\right] \\
& =\left[\begin{array}{cccccc}
1 & & & \\
0 & 1 & & \\
0.1 & \frac{-10}{69.97} & 1 & \\
0 & 0 & \frac{34.985}{55975} & 1
\end{array}\right]\left[\begin{array}{cccc}
1000 & & & \\
& 69.97 & & \\
& & \frac{559750}{6997} & \\
& & & .1
\end{array}\right]\left[\begin{array}{cccc}
1 & 0.1 & 0.5 & 0.1 \\
& 1 & \frac{69.96}{69.97} & 0 \\
& & 1 & 0 \\
& & & 1
\end{array}\right]
\end{aligned}
$$

and

$$
\begin{aligned}
& P_{1} \tilde{A} P_{1}^{T}=\left[\begin{array}{cccc}
1000 & 100 & 500 & 101 \\
0 & 69.97 & 69.96 & 0 \\
100 & 0 & 120 & 10 \\
0 & 0 & 0.05 & 0.1
\end{array}\right]
\end{aligned}
$$

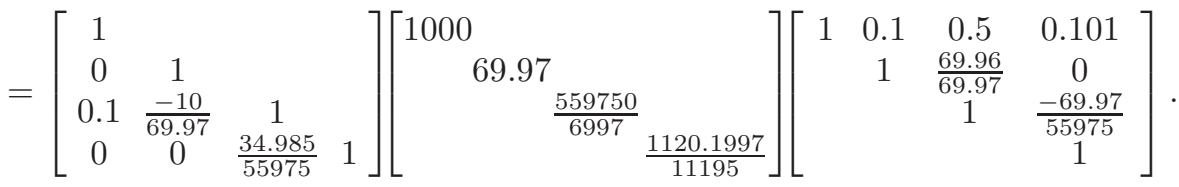

Observe that the $L$ factors of $P_{1} A P_{1}^{T}$ and $P_{1} \tilde{A} P_{1}^{T}$ are identical, the componentwise relative difference of the $D$ factors is smaller than $10^{-2}$, and the normwise difference of the $U$ factors is also smaller than $10^{-2}$ in the $\infty$-norm. This is in accordance with Theorem 3.2. In addition, the $L$ factor of $P_{1} A P_{1}^{T}$ is now column diagonally dominant.

We emphasize that this example shows how subtle is the dependence of the perturbation properties of the factor $L$ on the pivoting strategy: observe that the $L$ factor of the unpivoted matrix $A$ is also column diagonally dominant and that its $D$ and $U$ factors are very stable under perturbations of type (3.2). However, despite these favorable properties, the $L$ factor of $A$ is very sensitive. Finally, note also that 
in this example all of the $L$ factors of $A, P A P^{T}$, and $P_{1} A P_{1}^{T}$ are well-conditioned, since their condition numbers in the 1-norm are approximately equal to 4,4 , and 1.3 , respectively.

The rest of this section is devoted to proving Theorem 3.2. Clearly, as a consequence of Theorem 3.1, we only need to prove (3.3), but this requires considerable effort and the development of several auxiliary technical lemmas in advance. These lemmas may be also of interest for other purposes, and are presented in section 3.1 together with the proof of Theorem 3.2.

3.1. Auxiliary lemmas and proof of Theorem 3.2. The proofs of Theorem 3.1 in [14] and our Theorem 3.2 hinge on Theorem 2.5 and several results for determinants and minors of row diagonally dominant matrices that were proved in [14]. Given a matrix $A \in \mathbb{R}^{n \times n}$, the following will be used to denote some of its minors:

$$
g_{p q}^{(k+1)}:=\operatorname{det} A([1: k, p],[1: k, q])
$$

for $1 \leq k \leq n-1$ and $k+1 \leq p, q \leq n$. Note that all of the determinants appearing in Theorem 2.5 and (2.5) are particular cases of the determinants defined in (3.4) and, in particular, the entries of $L$ can be expressed as

$$
l_{i j}=\frac{g_{i j}^{(j)}}{g_{j j}^{(j)}}, \quad \text { where } \quad i \geq j .
$$

Then, the proof of part (c) of Theorem 3.1 in [14] is based on perturbation bounds first established for minors $g_{i j}^{(j)}$. Under perturbations of type (3.2), the variation of principal minors $g_{i i}^{(j)}$ is small relative to itself but for the nonprincipal minors $g_{i j}^{(j)}$ (with $i \neq j$ ), the variation can only be bounded to be small relative to $g_{i i}^{(j)}$. Applying the perturbation bounds for minors to (3.5) leads to a perturbation bound for $l_{i j}$ that is small relative to

$$
\frac{g_{i i}^{(j)}}{g_{j j}^{(j)}}=\frac{g_{i i}^{(j)} / g_{j-1, j-1}^{(j-1)}}{g_{j j}^{(j)} / g_{j-1, j-1}^{(j-1)}}=\frac{a_{i i}^{(j)}}{a_{j j}^{(j)}},
$$

where the second equality follows from (2.5). The absolute value of this term is bounded by 1 with the complete pivoting strategy but may be arbitrarily large with the column diagonal dominance pivoting strategy. To prove the stronger bound of Theorem 3.2, we shall prove in Lemma 3.11 that the variation of the nonprincipal minors $g_{i j}^{(j)}$ is small relative to $\left(v_{i}^{(j)}+\left|a_{i j}^{(j)}\right|\right) g_{j-1, j-1}^{(j-1)}$. Then, the perturbation of $l_{i j}$ is small relative to $\left(v_{i}^{(j)}+\left|a_{i j}^{(j)}\right|\right) / a_{j j}^{(j)}$, which will be proved in Lemma 3.12 to be bounded by $n-j$ for the column diagonal dominance pivoting strategy. Thus, $l_{i j}$ and hence $L$ have a small perturbation bound as shown in Theorem 3.2.

The proof of Lemma 3.11 on the strong perturbation bound for the nonprincipal minors $g_{i j}^{(j)}$ turns out to be difficult. While we shall follow an approach similar to [14] in dealing with variations of minors, the proof involves a judicious construction of an auxiliary matrix and its corresponding perturbation that no longer satisfies the standard perturbation condition (3.2). As a result, we need to consider auxiliary perturbations that are more general than those in (3.2). More precisely, for a fixed $p$, the $p$ th column of $A$ will be perturbed in the particular way appearing in (3.7), which corresponds to larger perturbations than those in (3.2) for the off-diagonal entries in 
the $p$ th column. Specifically, we consider matrices $A=\left[a_{i j}\right]=\mathcal{D}\left(A_{D}, v\right) \in \mathbb{R}^{n \times n}$, with $v \geq 0$, and $\tilde{A}=\left[\tilde{a}_{i j}\right]=\mathcal{D}\left(\tilde{A}_{D}, \tilde{v}\right) \in \mathbb{R}^{n \times n}$ that satisfy, for $0 \leq \epsilon<1$,

$$
\begin{aligned}
|\tilde{v}-v| & \leq \epsilon v \\
\left|\tilde{a}_{i p}-a_{i p}\right| & \leq \epsilon\left(v_{i}+\left|a_{i p}\right|\right) \quad \text { for } i \in\{1: n\} \backslash\{p\}, \quad \text { and } \\
\left|\tilde{a}_{i j}-a_{i j}\right| & \leq \epsilon\left|a_{i j}\right| \quad \text { for } i \neq j, i \in\{1: n\}, j \in\{1: n\} \backslash\{p\} .
\end{aligned}
$$

This generalized perturbation can be equivalently expressed as

$$
\begin{aligned}
\tilde{v}_{i} & =v_{i}\left(1+\phi_{i}\right) \text { with }\left|\phi_{i}\right| \leq \epsilon \text { for } i \in\{1: n\} \\
\tilde{a}_{i p} & =a_{i p}\left(1+\phi_{i p}^{\prime}\right)+\phi_{i p} v_{i} \text { with }\left|\phi_{i p}\right| \leq \epsilon, \phi_{i p}^{\prime}=\phi_{i p} \operatorname{sign}\left(a_{i p}\right) \text { for } i \in\{1: n\} \backslash\{p\} \\
\tilde{a}_{i j} & =a_{i j}\left(1+\phi_{i j}\right) \text { with }\left|\phi_{i j}\right| \leq \epsilon \text { for } i \neq j, i \in\{1: n\}, j \in\{1: n\} \backslash\{p\}
\end{aligned}
$$

Observe that from (3.6), we obtain again that $\tilde{v} \geq 0$ holds and, so, this generalized perturbation also preserves the row diagonally dominant structure, as well as the nonnegativity of the diagonal entries.

We note that in the first part of this section the perturbation parameter $\epsilon$ can be considered to satisfy $0 \leq \epsilon<1$ and $A$ can be any row diagonally dominant matrix. Only at the end of this section will it be imposed that $A$ is arranged for column diagonal dominance pivoting.

Before presenting new results, we state for completeness some identities and inequalities from [14] concerning determinants and minors of diagonally dominant matrices.

Lemma 3.4 (see [14, Lemma 1]). Let $A=\mathcal{D}\left(A_{D}, v\right) \in \mathbb{R}^{n \times n}$ be such that $v \geq 0$. Denote the algebraic cofactors of $A$ by $C_{i j}:=(-1)^{i+j} \operatorname{det} A\left(i^{\prime}, j^{\prime}\right), 1 \leq i, j \leq n$. Then

$$
\operatorname{det} A=v_{i} C_{i i}+\sum_{j \neq i}\left(\left|a_{i j}\right| C_{i i}+a_{i j} C_{i j}\right), \quad i=1, \ldots, n
$$

with $v_{i} C_{i i} \geq 0$ and $\left|a_{i j}\right| C_{i i}+a_{i j} C_{i j} \geq 0$ for $j \neq i$.

Lemma 3.5 (see [14, Lemma 6]). Let $A=\mathcal{D}\left(A_{D}, v\right) \in \mathbb{R}^{n \times n}$ be such that $v \geq 0$. For $k=1, \ldots, n-2, p \neq q$, and $k+1 \leq p, q \leq n$, let $G_{i j}$ be the algebraic cofactor of $A([1: k, p],[1: k, q])$ for the entry $a_{i j}$. Then the minors defined in (3.4) satisfy

$$
\begin{aligned}
g_{p q}^{(k+1)} & =a_{p 1} G_{p 1}+\cdots+a_{p k} G_{p k}+a_{p q} G_{p q} \\
2 g_{p p}^{(k+1)} & \geq\left|a_{p 1} G_{p 1}\right|+\cdots+\left|a_{p k} G_{p k}\right|+\left|a_{p q} G_{p q}\right|
\end{aligned}
$$

and, for $1 \leq i \leq k$,

$$
\begin{aligned}
g_{p q}^{(k+1)} & =\left(v_{i}+\sum_{j \notin\{1, \ldots, k, q\}}\left|a_{i j}\right|\right) G_{i i}+\sum_{j \in\{1, \ldots, k, q\} \backslash\{i\}}\left(a_{i j} G_{i j}+\left|a_{i j}\right| G_{i i}\right), \\
2 g_{p p}^{(k+1)} & \geq\left(v_{i}+\sum_{j \notin\{1, \ldots, k, q\}}\left|a_{i j}\right|\right)\left|G_{i i}\right|+\sum_{j \in\{1, \ldots, k, q\} \backslash\{i\}}\left|a_{i j} G_{i j}+\right| a_{i j}\left|G_{i i}\right| .
\end{aligned}
$$

Our first lemma generalizes [14, Lemma 3] by replacing the standard perturbation condition (3.2) with the generalized perturbation conditions defined in (3.6)-(3.8).

Copyright (C) by SIAM. Unauthorized reproduction of this article is prohibited. 
Lemma 3.6. Let $A=\mathcal{D}\left(A_{D}, v\right) \in \mathbb{R}^{n \times n}$ be such that $v \geq 0$ and let $\tilde{A}=$ $\mathcal{D}\left(\tilde{A}_{D}, \tilde{v}\right) \in \mathbb{R}^{n \times n}$ satisfy (3.6)-(3.8) with $0 \leq \epsilon<1$. Suppose $\tilde{A}^{[i]}=\mathcal{D}\left(\tilde{A}_{D}^{[i]}, \tilde{v}^{[i]}\right) \in$ $\mathbb{R}^{n \times n}$ is a matrix that differs from $A$ in only the ith row and whose ith row is the same as the ith row of $\tilde{A}$. Then

$$
\operatorname{det} \tilde{A}^{[i]}=(\operatorname{det} A)\left(1+\eta_{i}\right), \text { where }\left|\eta_{i}\right| \leq 3 \epsilon \text {. }
$$

Proof. Let $A=\left[a_{j k}\right], v=\left[v_{j}\right], \tilde{A}=\left[\tilde{a}_{j k}\right]$, and $\tilde{v}=\left[\tilde{v}_{j}\right]$. We consider the cofactor expansion of $\operatorname{det} \tilde{A}^{[i]}$ across row $i$. Let $\tilde{C}_{i j}$ be the algebraic cofactor of $\tilde{A}^{[i]}$ corresponding to $\tilde{a}_{i j}^{[i]}$ and $C_{i j}$ be the algebraic cofactor of $A$ corresponding to $a_{i j}$. Then, $\tilde{C}_{i j}=C_{i j}$. We need to discuss two cases separately.

Case 1: $i=p$. By Lemma 3.4, we have

$$
\operatorname{det} \tilde{A}^{[p]}=\tilde{v}_{p} \tilde{C}_{p p}+\sum_{j \neq p}\left(\left|\tilde{a}_{p j}\right| \tilde{C}_{p p}+\tilde{a}_{p j} \tilde{C}_{p j}\right)=\tilde{v}_{p} C_{p p}+\sum_{j \neq p}\left(\left|\tilde{a}_{p j}\right| C_{p p}+\tilde{a}_{p j} C_{p j}\right)
$$

and a similar equation for $\operatorname{det} A$. Lemma 3.4 further implies that $v_{p} C_{p p} \geq 0$ and $\left|a_{p j}\right| C_{p p}+a_{p j} C_{p j} \geq 0$. Using (3.9) and (3.11), we obtain

$$
\operatorname{det} \tilde{A}^{[p]}=\operatorname{det} A+\phi_{p} v_{p} C_{p p}+\sum_{j \neq p} \phi_{p j}\left(\left|a_{p j}\right| C_{p p}+a_{p j} C_{p j}\right)
$$

It follows that

$$
\begin{aligned}
\left|\operatorname{det} \tilde{A}^{[p]}-\operatorname{det} A\right| & \leq\left|\phi_{p}\right| v_{p} C_{p p}+\sum_{j \neq p}\left|\phi_{p j}\right|\left(\left|a_{p j}\right| C_{p p}+a_{p j} C_{p j}\right) \\
& \leq \epsilon v_{p} C_{p p}+\sum_{j \neq p} \epsilon\left(\left|a_{p j}\right| C_{p p}+a_{p j} C_{p j}\right) \\
& =\epsilon \operatorname{det} A,
\end{aligned}
$$

which proves the lemma for this case.

Case 2: $i \neq p$. We again use Lemma 3.4, (3.9), (3.10), and (3.11) to obtain

$$
\begin{aligned}
\operatorname{det} \tilde{A}^{[i]}= & \tilde{v}_{i} \tilde{C}_{i i}+\sum_{j \neq i}\left(\left|\tilde{a}_{i j}\right| \tilde{C}_{i i}+\tilde{a}_{i j} \tilde{C}_{i j}\right) \\
= & \tilde{v}_{i} C_{i i}+\left|\tilde{a}_{i p}\right| C_{i i}+\tilde{a}_{i p} C_{i p}+\sum_{j \neq i, p}\left(\left|\tilde{a}_{i j}\right| C_{i i}+\tilde{a}_{i j} C_{i j}\right) \\
= & v_{i} C_{i i}+v_{i} \phi_{i} C_{i i}+\left|\tilde{a}_{i p}\right| C_{i i}+a_{i p} C_{i p}+a_{i p} \phi_{i p}^{\prime} C_{i p}+\phi_{i p} v_{i} C_{i p} \\
& +\sum_{j \neq i, p}\left(\left|a_{i j}\right| C_{i i}+a_{i j} C_{i j}\right)+\sum_{j \neq i, p} \phi_{i j}\left(\left|a_{i j}\right| C_{i i}+a_{i j} C_{i j}\right)
\end{aligned}
$$

From (3.10), we have

$$
\left|a_{i p}\right|\left(1+\phi_{i p}^{\prime}\right)-\epsilon v_{i} \leq\left|\tilde{a}_{i p}\right| \leq\left|a_{i p}\right|\left(1+\phi_{i p}^{\prime}\right)+\epsilon v_{i},
$$

Copyright $@$ by SIAM. Unauthorized reproduction of this article is prohibited. 
and hence, from (3.17), Lemma 3.4, and $\left|C_{i p}\right| \leq C_{i i}$ (see Theorem 2.3(e)),

$$
\begin{aligned}
\operatorname{det} \tilde{A}^{[i]} \geq & v_{i} C_{i i}+v_{i} \phi_{i} C_{i i}+\left|a_{i p}\right| C_{i i}+\left|a_{i p}\right| \phi_{i p}^{\prime} C_{i i}-\epsilon v_{i} C_{i i}+a_{i p} C_{i p}+a_{i p} \phi_{i p}^{\prime} C_{i p} \\
& +\phi_{i p} v_{i} C_{i p}+\sum_{j \neq i, p}\left(\left|a_{i j}\right| C_{i i}+a_{i j} C_{i j}\right)+\sum_{j \neq i, p} \phi_{i j}\left(\left|a_{i j}\right| C_{i i}+a_{i j} C_{i j}\right) \\
= & \operatorname{det} A+\phi_{i} v_{i} C_{i i}+\phi_{i p}^{\prime}\left(\left|a_{i p}\right| C_{i i}+a_{i p} C_{i p}\right)-\epsilon v_{i} C_{i i}+\phi_{i p} v_{i} C_{i p} \\
& +\sum_{j \neq i, p} \phi_{i j}\left(\left|a_{i j}\right| C_{i i}+a_{i j} C_{i j}\right) \\
\geq & \operatorname{det} A-\epsilon v_{i} C_{i i}-\epsilon\left(\left|a_{i p}\right| C_{i i}+a_{i p} C_{i p}\right)-\epsilon v_{i} C_{i i}-\epsilon v_{i} C_{i i} \\
& -\epsilon \sum_{j \neq i, p}\left(\left|a_{i j}\right| C_{i i}+a_{i j} C_{i j}\right) \\
= & \operatorname{det} A-3 \epsilon v_{i} C_{i i}-\epsilon \sum_{j \neq i}\left(\left|a_{i j}\right| C_{i i}+a_{i j} C_{i j}\right) \\
\geq & \operatorname{det} A-3 \epsilon \operatorname{det} A .
\end{aligned}
$$

Similarly,

$$
\begin{aligned}
\operatorname{det} \tilde{A}^{[i]} \leq & v_{i} C_{i i}+v_{i} \phi_{i} C_{i i}+\left|a_{i p}\right| C_{i i}+\left|a_{i p}\right| \phi_{i p}^{\prime} C_{i i}+\epsilon v_{i} C_{i i}+a_{i p} C_{i p}+a_{i p} \phi_{i p}^{\prime} C_{i p} \\
& +\phi_{i p} v_{i} C_{i p}+\sum_{j \neq i, p}\left(\left|a_{i j}\right| C_{i i}+a_{i j} C_{i j}\right)+\sum_{j \neq i, p} \phi_{i j}\left(\left|a_{i j}\right| C_{i i}+a_{i j} C_{i j}\right) \\
\leq & \operatorname{det} A+\epsilon v_{i} C_{i i}+\epsilon\left(\left|a_{i p}\right| C_{i i}+a_{i p} C_{i p}\right)+\epsilon v_{i} C_{i i}+\epsilon v_{i}\left|C_{i p}\right| \\
& +\epsilon \sum_{j \neq i, p}\left(\left|a_{i j}\right| C_{i i}+a_{i j} C_{i j}\right) \\
\leq & \operatorname{det} A+3 \epsilon v_{i} C_{i i}+\epsilon \sum_{j \neq i}\left(\left|a_{i j}\right| C_{i i}+a_{i j} C_{i j}\right) \\
\leq & \operatorname{det} A+3 \epsilon \operatorname{det} A .
\end{aligned}
$$

Thus,

$$
\left|\operatorname{det} \tilde{A}^{[i]}-\operatorname{det} A\right| \leq 3 \epsilon \operatorname{det} A,
$$

which proves the lemma.

Lemma 3.7 below uses Lemma 3.6 to present a similar perturbation bound for the principal minors of a row diagonally dominant matrix under the structured perturbations defined in (3.6)-(3.8). It generalizes [14, Lemma 4] which has to assume the standard perturbation condition (3.2).

Lemma 3.7. Let $A=\mathcal{D}\left(A_{D}, v\right) \in \mathbb{R}^{n \times n}$ be such that $v \geq 0$ and let $\tilde{A}=$ $\mathcal{D}\left(\tilde{A}_{D}, \tilde{v}\right) \in \mathbb{R}^{n \times n}$ satisfy (3.6)-(3.8) with $0 \leq \epsilon<1 / 2$. Suppose $\tilde{A}^{[i]}=\mathcal{D}\left(\tilde{A}_{D}^{[i]}, \tilde{v}^{[i]}\right)$ $\in \mathbb{R}^{n \times n}$ is a matrix that differs from $A$ in only the ith row and whose ith row is the same as the $i$ th row of $\tilde{A}$. Let $1 \leq i_{1}<i_{2}<\cdots<i_{q} \leq n$ and $\alpha=\left\{i_{1}, i_{2}, \ldots, i_{q}\right\}$, and recall that $A(\alpha, \alpha)$ denotes the principal submatrix of $A$ that lies in rows and columns indexed by $\alpha$. Then

$$
\operatorname{det} \tilde{A}^{[i]}(\alpha, \alpha)= \begin{cases}\operatorname{det} A(\alpha, \alpha) & \text { if } i \notin \alpha, \\ (\operatorname{det} A(\alpha, \alpha))\left(1+\delta_{i}^{(\alpha)}\right) & \text { if } i \in \alpha,\end{cases}
$$

Copyright () by SIAM. Unauthorized reproduction of this article is prohibited. 
where $\left|\delta_{i}^{(\alpha)}\right| \leq 6 \epsilon$ if $p \notin \alpha$ and $\left|\delta_{i}^{(\alpha)}\right| \leq 3 \epsilon$ if $p \in \alpha$. Furthermore,

$$
\operatorname{det} \tilde{A}(\alpha, \alpha)=(\operatorname{det} A(\alpha, \alpha))\left(1+\eta_{1}^{(\alpha)}\right) \cdots\left(1+\eta_{q}^{(\alpha)}\right),
$$

where $\left|\eta_{k}^{(\alpha)}\right| \leq 6 \epsilon$ if $p \notin \alpha$ and $\left|\eta_{k}^{(\alpha)}\right| \leq 3 \epsilon$ if $p \in \alpha$, for $k=1, \ldots, q$.

Proof. We prove first (3.19). Assume $i \in \alpha$, otherwise the result is trivial. Since $A=\left[a_{j k}\right]$ and $\tilde{A}^{[i]}=\left[\tilde{a}_{j k}^{[i]}\right]$ are row diagonally dominant with nonnegative diagonal entries, then so are $A(\alpha, \alpha)$ and $\tilde{A}^{[i]}(\alpha, \alpha)$. Hence, we can parameterize them in terms of their diagonally dominant parts and off-diagonal entries. Let

$$
A(\alpha, \alpha)=\mathcal{D}\left(A_{D}(\alpha, \alpha), w\right) \quad \text { and } \quad \tilde{A}^{[i]}(\alpha, \alpha)=\mathcal{D}\left(\tilde{A}_{D}^{[i]}(\alpha, \alpha), \tilde{w}^{[i]}\right),
$$

where $w=\left[w_{j}\right], \tilde{w}^{[i]}=\left[\tilde{w}_{j}^{[i]}\right] \in \mathbb{R}^{q}$. For simplicity, the entries and diagonally dominant parts of $A(\alpha, \alpha)$ and $\tilde{A}^{[i]}(\alpha, \alpha)$ are indexed with the indices $i_{1}, i_{2}, \ldots, i_{q}$ in $\alpha$. Let $v=\left[v_{j}\right]$ and $\tilde{v}^{[i]}=\left[\tilde{v}_{j}^{[i]}\right]$. Let us compare the diagonally dominant parts of $A(\alpha, \alpha)$ and $\tilde{A}^{[i]}(\alpha, \alpha)$. To this purpose observe that $w_{j}=\tilde{w}_{j}^{[i]}$ if $j \in \alpha \backslash\{i\}$,

$$
w_{i}=a_{i i}-\sum_{j \in \alpha \backslash\{i\}}\left|a_{i j}\right|=\left(v_{i}+\sum_{j \neq i}\left|a_{i j}\right|\right)-\sum_{j \in \alpha \backslash\{i\}}\left|a_{i j}\right|=v_{i}+\sum_{j \notin \alpha}\left|a_{i j}\right|,
$$

and, similarly, $\tilde{w}_{i}^{[i]}=\tilde{v}_{i}^{[i]}+\sum_{j \notin \alpha}\left|\tilde{a}_{i j}^{[i]}\right|$. Thus, we have

$$
\left|\tilde{w}_{i}^{[i]}-w_{i}\right|=\left|\tilde{v}_{i}^{[i]}-v_{i}+\sum_{j \notin \alpha}\left(\left|\tilde{a}_{i j}^{[i]}\right|-\left|a_{i j}\right|\right)\right| \leq\left|\tilde{v}_{i}^{[i]}-v_{i}\right|+\sum_{j \notin \alpha}\left|\tilde{a}_{i j}^{[i]}-a_{i j}\right| .
$$

If $p \in \alpha$, then (3.6) and (3.8) imply

$$
\left|\tilde{w}_{i}^{[i]}-w_{i}\right| \leq \epsilon v_{i}+\sum_{j \notin \alpha} \epsilon\left|a_{i j}\right|=\epsilon\left(v_{i}+\sum_{j \notin \alpha}\left|a_{i j}\right|\right)=\epsilon w_{i} .
$$

Since $w_{i} \geq v_{i}$, by (3.21), the off-diagonal entries of $\tilde{A}^{[i]}(\alpha, \alpha)$ and $A(\alpha, \alpha)$ satisfy conditions (3.7)-(3.8) for their parameters. Therefore, we can apply Lemma 3.6 to $\tilde{A}^{[i]}(\alpha, \alpha)$ and $A(\alpha, \alpha)$ to obtain that, if $p \in \alpha$, then

$$
\operatorname{det} \tilde{A}^{[i]}(\alpha, \alpha)=(\operatorname{det} A(\alpha, \alpha))\left(1+\delta_{i}^{(\alpha)}\right)
$$

with $\left|\delta_{i}^{(\alpha)}\right| \leq 3 \epsilon$. If $p \notin \alpha$, then, from (3.22) and (3.6)-(3.8), we get

$$
\begin{aligned}
\left|\tilde{w}_{i}^{[i]}-w_{i}\right| & \leq\left|\tilde{v}_{i}^{[i]}-v_{i}\right|+\sum_{j \notin \alpha, j \neq p}\left|\tilde{a}_{i j}^{[i]}-a_{i j}\right|+\left|\tilde{a}_{i p}^{[i]}-a_{i p}\right| \\
& \leq \epsilon v_{i}+\sum_{j \notin \alpha, j \neq p} \epsilon\left|a_{i j}\right|+\epsilon\left(v_{i}+\left|a_{i p}\right|\right) \\
& =2 \epsilon v_{i}+\sum_{j \notin \alpha} \epsilon\left|a_{i j}\right| \leq 2 \epsilon w_{i} .
\end{aligned}
$$

Copyright $@$ by SIAM. Unauthorized reproduction of this article is prohibited. 
Again, the off-diagonal entries of $\tilde{A}^{[i]}(\alpha, \alpha)$ and $A(\alpha, \alpha)$ satisfy (3.7)-(3.8) for their parameters. So, we can apply Lemma 3.6 to $\tilde{A}^{[i]}(\alpha, \alpha)$ and $A(\alpha, \alpha)$, but this time with $\epsilon$ replaced by $2 \epsilon$, which requires $2 \epsilon<1$, to obtain

$$
\operatorname{det} \tilde{A}^{[i]}(\alpha, \alpha)=(\operatorname{det} A(\alpha, \alpha))\left(1+\delta_{i}^{(\alpha)}\right)
$$

with $\left|\delta_{i}^{(\alpha)}\right| \leq 6 \epsilon$, for $p \notin \alpha$. This proves (3.19).

Finally, we prove (3.20). To this purpose, consider that the perturbed submatrix $\tilde{A}(\alpha, \alpha)$ can be obtained from $A(\alpha, \alpha)$ by a sequence of "only one row" at a time perturbations. By (3.19), each of these "only one row" perturbations produces a determinant that is equal to the determinant before the perturbation times a factor $1+\eta$, with $|\eta| \leq 6 \epsilon$ if $p \notin \alpha$ and $|\eta| \leq 3 \epsilon$ if $p \in \alpha$.

The next lemma considers the variation of certain nonprincipal minors of a row diagonally dominant matrix $A$ under the structured perturbations defined in (3.6)(3.8). It generalizes [14, Lemma 7$]$ which assumes the standard perturbation condition (3.2). In this lemma, observe that the last row of these minors corresponds precisely to the fixed index $p$ appearing in (3.6)-(3.8). Also, the minors of $A$ that are of interest are denoted as in (3.4), that is,

$$
g_{p q}^{(k+1)}=\operatorname{det} A([1: k, p],[1: k, q])
$$

for $1 \leq k \leq n-1$ and $k+1 \leq p, q \leq n$. We also denote by $\left(\tilde{g}^{[i]}\right)_{p q}^{(k+1)}$ and $\tilde{g}_{p q}^{(k+1)}$ the corresponding minors of the perturbed matrices $\tilde{A}^{[i]}$ and $\tilde{A}$, respectively, which were defined in Lemmas 3.6 and 3.7.

LEMMA 3.8. Let $A=\mathcal{D}\left(A_{D}, v\right) \in \mathbb{R}^{n \times n}$ be such that $v \geq 0$ and let $\tilde{A}=$ $\mathcal{D}\left(\tilde{A}_{D}, \tilde{v}\right) \in \mathbb{R}^{n \times n}$ satisfy (3.6)-(3.8) with $0 \leq \epsilon<1 / 2$. Suppose $\tilde{A}^{[i]}=\mathcal{D}\left(\tilde{A}_{D}^{[i]}, \tilde{v}^{[i]}\right)$ $\in \mathbb{R}^{n \times n}$ is a matrix that differs from $A$ in only the ith row and whose ith row is the same as the ith row of $\tilde{A}$. Let $1 \leq k \leq n-2, k+1 \leq p, q \leq n$, and $p \neq q$, where $p$ is the fixed index in (3.6)-(3.8). Then, the following statements hold for the minors in (3.23):
(a) $\left|\left(\tilde{g}^{[i]}\right)_{p q}^{(k+1)}-g_{p q}^{(k+1)}\right| \leq \begin{cases}0 & \text { if } i \notin\{1: k, p\} \\ 4 \epsilon g_{p p}^{(k+1)} & \text { if } i \in\{1: k, p\}\end{cases}$
(b) $\left|\tilde{g}_{p q}^{(k+1)}-g_{p q}^{(k+1)}\right| \leq \frac{4}{3}\left((1+3 \epsilon)^{k+1}-1\right) g_{p p}^{(k+1)}$.

Proof. Let $A=\left[a_{j k}\right], \tilde{A}^{[i]}=\left[\tilde{a}_{j k}^{[i]}\right], \tilde{A}=\left[\tilde{a}_{j k}\right], v=\left[v_{j}\right], \tilde{v}^{[i]}=\left[\tilde{v}_{j}^{[i]}\right]$, and $\tilde{v}=\left[\tilde{v}_{j}\right]$. We prove first part (a) for $i \in\{1: k, p\}$, as the case $i \notin\{1: k, p\}$ is trivial. For $j \in\{1: k, q\}$, let $G_{i j}$ be the algebraic cofactor of $A([1: k, p],[1: k, q])$ for the entry $a_{i j}$, and note that this is also the algebraic cofactor of $\tilde{A}^{[i]}([1: k, p],[1: k, q])$ for the entry $\tilde{a}_{i j}^{[i]}$.

If $1 \leq i \leq k$, applying Lemma 3.5 yields

$$
\begin{aligned}
\left(\tilde{g}^{[i]}\right)_{p q}^{(k+1)} & =\left(\tilde{v}_{i}^{[i]}+\sum_{j \notin\{1: k, q\}}\left|\tilde{a}_{i j}^{[i]}\right|\right) G_{i i}+\sum_{j \in\{1: k, q\} \backslash\{i\}}\left(\tilde{a}_{i j}^{[i]} G_{i j}+\left|\tilde{a}_{i j}^{[i]}\right| G_{i i}\right) \\
& =\left(\tilde{v}_{i}+\sum_{j \notin\{1: k, p, q\}}\left|\tilde{a}_{i j}\right|\right) G_{i i}+\left|\tilde{a}_{i p}\right| G_{i i}+\sum_{j \in\{1: k, q\} \backslash\{i\}}\left(\tilde{a}_{i j} G_{i j}+\left|\tilde{a}_{i j}\right| G_{i i}\right) .
\end{aligned}
$$

Copyright $\odot$ by SIAM. Unauthorized reproduction of this article is prohibited. 
Similarly, we have

$$
g_{p q}^{(k+1)}=\left(v_{i}+\sum_{j \notin\{1: k, p, q\}}\left|a_{i j}\right|\right) G_{i i}+\left|a_{i p}\right| G_{i i}+\sum_{j \in\{1: k, q\} \backslash\{i\}}\left(a_{i j} G_{i j}+\left|a_{i j}\right| G_{i i}\right) .
$$

Using (3.9) and (3.11), we can write

$$
\begin{aligned}
\left(\tilde{g}^{[i]}\right)_{p q}^{(k+1)}= & g_{p q}^{(k+1)}+\left(v_{i} \phi_{i}+\sum_{j \notin\{1: k, p, q\}} \phi_{i j}\left|a_{i j}\right|\right) G_{i i} \\
& +\sum_{j \in\{1: k, q\} \backslash\{i\}} \phi_{i j}\left(a_{i j} G_{i j}+\left|a_{i j}\right| G_{i i}\right)+\left(\left|\tilde{a}_{i p}\right|-\left|a_{i p}\right|\right) G_{i i} .
\end{aligned}
$$

Therefore, using (3.7), we get

$$
\begin{aligned}
& \left|\left(\tilde{g}^{[i]}\right)_{p q}^{(k+1)}-g_{p q}^{(k+1)}\right| \\
& \leq \epsilon\left[\left(v_{i}+\sum_{j \notin\{1: k, p, q\}}\left|a_{i j}\right|\right)\left|G_{i i}\right|\right. \\
& \left.\quad+\sum_{j \in\{1: k, q\} \backslash\{i\}}\left|a_{i j} G_{i j}+\right| a_{i j}\left|G_{i i}\right|+\left|a_{i p}\right|\left|G_{i i}\right|+v_{i}\left|G_{i i}\right|\right] \\
& \leq 2 \epsilon\left[\left(v_{i}+\sum_{j \notin\{1: k, q\}}\left|a_{i j}\right|\right)\left|G_{i i}\right|+\sum_{j \in\{1: k, q\} \backslash\{i\}}\left|a_{i j} G_{i j}+\right| a_{i j}\left|G_{i i}\right|\right] \\
& \leq 4 \epsilon g_{p p}^{(k+1)},
\end{aligned}
$$

where Lemma 3.5 has been used in the last inequality. This proves part (a) for $1 \leq i \leq k$.

Now, for $i=p$, use Lemma 3.5 and (3.11) to obtain

$$
\begin{aligned}
\left(\tilde{g}^{[p]}\right)_{p q}^{(k+1)} & =\sum_{j \in\{1: k, q\}} \tilde{a}_{p j}^{[p]} G_{p j}=\sum_{j \in\{1: k, q\}} a_{p j}\left(1+\phi_{p j}\right) G_{p j} \\
& =\sum_{j \in\{1: k, q\}} a_{p j} G_{p j}+\sum_{j \in\{1: k, q\}} \phi_{p j} a_{p j} G_{p j}=g_{p q}^{(k+1)}+\sum_{j \in\{1: k, q\}} \phi_{p j} a_{p j} G_{p j} .
\end{aligned}
$$

Thus,

$$
\left|\left(\tilde{g}^{[p]}\right)_{p q}^{(k+1)}-g_{p q}^{(k+1)}\right| \leq \epsilon \sum_{j \in\{1: k, q\}}\left|a_{p j} G_{p j}\right| \leq \epsilon\left(2 g_{p p}^{(k+1)}\right),
$$

where Lemma 3.5 has been used again in the last inequality. This proves part (a) for $i=p$ and completes the whole proof of this part.

For part (b), consider obtaining $\tilde{A}$ from $A$ by a sequence of only one row at a time perturbations. Note that each matrix in this sequence is row diagonally dominant with nonnegative diagonals. The variation in $g_{p q}^{(k+1)}$ is a consequence only of the

Copyright (? by SIAM. Unauthorized reproduction of this article is prohibited. 
perturbations of rows with indices in $\{1: k, p\}$. Let $\alpha$ be a subset of $\{1: k, p\}$ and denote by $\left(\tilde{g}^{\alpha}\right)_{p q}^{(k+1)}$ the minor corresponding to a matrix obtained from $A$ through perturbations in the rows with indices in $\alpha$ only. Thus

$$
\begin{aligned}
\left|\tilde{g}_{p q}^{(k+1)}-g_{p q}^{(k+1)}\right| & =\left|\left(\tilde{g}^{\{1: k, p\}}\right)_{p q}^{(k+1)}-g_{p q}^{(k+1)}\right| \\
& \leq\left|\left(\tilde{g}^{\{1: k, p\}}\right)_{p q}^{(k+1)}-\left(\tilde{g}^{\{1: k\}}\right)_{p q}^{(k+1)}\right|+\cdots+\left|\left(\tilde{g}^{\{1\}}\right)_{p q}^{(k+1)}-g_{p q}^{(k+1)}\right| .
\end{aligned}
$$

Apply part (a) to each term in this sum to obtain,

$$
\left|\tilde{g}_{p q}^{(k+1)}-g_{p q}^{(k+1)}\right| \leq 4 \epsilon\left[\left(\tilde{g}^{\{1: k\}}\right)_{p p}^{(k+1)}+\cdots+g_{p p}^{(k+1)}\right]
$$

and then apply Lemma 3.7 to each term in the sum above to get

$$
\left|\tilde{g}_{p q}^{(k+1)}-g_{p q}^{(k+1)}\right| \leq 4 \epsilon\left[(1+3 \epsilon)^{k}+\cdots+1\right] g_{p p}^{(k+1)}=\frac{4}{3}\left((1+3 \epsilon)^{k+1}-1\right) g_{p p}^{(k+1)} .
$$

Lemma 3.11 later establishes a new perturbation bound for minors of row diagonally dominant matrices $A$ and $\tilde{A}$ satisfying the standard perturbation (3.2). It is the key lemma towards the proof of our main result, i.e., Theorem 3.2. Its proof involves construction from $A$ and $\tilde{A}$, via some elementary column operations, two new row diagonally dominant matrices with nonnegative diagonals $B$ and $\tilde{B} \in \mathbb{R}^{(k+2) \times(k+2)}$. We first show in Lemma 3.9 below, after considerable effort, that $B$ and $\widetilde{B}$ satisfy the generalized perturbation conditions (3.6)-(3.8). We warn the reader that the proof of Theorem 3.2 relies on applying Lemma 3.8 to the matrices $B$ and $\tilde{B}$. As $B$ is constructed from $A$, we label the $k+2$ rows and columns of $B$ using the indices $\{1: k, p, q\}$. While not traditional, this labeling is useful because we can easily compare entries in $B$ to entries in $A$. Thus, the $(k+1)$ th row and column of $B$ correspond to the $p$ th row and column of $A$ and similarly the $(k+2)$ nd row and column of $B$ correspond to the $q$ th row and column of $A$. Note also that in Lemma 3.9 the condition $\epsilon<1 / 5$ is imposed with the purpose only of guaranteeing $\delta<1 / 2$ in (3.29)-(3.31), which is necessary to apply Lemma 3.8 to $B$ and $\tilde{B}$.

Lemma 3.9. Let $A=\left[a_{i j}\right]=\mathcal{D}\left(A_{D}, v\right) \in \mathbb{R}^{n \times n}$ be such that $v \geq 0$ and let $\tilde{A}=\left[\tilde{a}_{i j}\right]=\mathcal{D}\left(\tilde{A}_{D}, \tilde{v}\right) \in \mathbb{R}^{n \times n}$ be a matrix that satisfies

$$
|\tilde{v}-v| \leq \epsilon v \quad \text { and } \quad\left|\tilde{A}_{D}-A_{D}\right| \leq \epsilon\left|A_{D}\right| \quad \text { for some } 0 \leq \epsilon<\frac{1}{5} .
$$

Let $A^{(k+1)}=\left[a_{i j}^{(k+1)}\right] \in \mathbb{R}^{n \times n}$ be the matrix obtained after $k$ stages of Gaussian elimination have been performed on $A$ and, for a fixed index $p$ such that $k+1 \leq p \leq n$, let $s_{j}=\operatorname{sign}\left(a_{p j}^{(k+1)}\right)$ for $j=1, \ldots, n$. Let $1 \leq k \leq n-2$ and let $B=\left[b_{i j}\right] \in \mathbb{R}^{(k+2) \times(k+2)}$ be a matrix whose rows and columns are indexed by $i, j \in\{1,2, \ldots, k, p, q\}$ and is defined as follows:

$$
\begin{cases}b_{i j}=a_{i j} & \text { for } i \in\{1: k, p, q\} \text { and } j \in\{1: k, q\}, \\ b_{i p}=a_{i p}-\sum_{j \notin\{1: k, p, q\}} s_{j} a_{i j} & \text { for } i \in\{1: k, p, q\} .\end{cases}
$$

Similarly, let $\tilde{B}=\left[\tilde{b}_{i j}\right] \in \mathbb{R}^{(k+2) \times(k+2)}$ be defined by

$$
\begin{cases}\tilde{b}_{i j}=\tilde{a}_{i j} & \text { for } i \in\{1: k, p, q\} \text { and } j \in\{1: k, q\}, \\ \tilde{b}_{i p}=\tilde{a}_{i p}-\sum_{j \notin\{1: k, p, q\}} s_{j} \tilde{a}_{i j} & \text { for } i \in\{1: k, p, q\} .\end{cases}
$$

Copyright ( by SIAM. Unauthorized reproduction of this article is prohibited. 
Then $B$ and $\tilde{B}$ are row diagonally dominant matrices with nonnegative diagonal entries. In addition, $B$ and $\tilde{B}$ can be parameterized as $B=\mathcal{D}\left(B_{D}, w\right)$ and $\tilde{B}=$ $\mathcal{D}\left(\tilde{B}_{D}, \tilde{w}\right)$ and satisfy

$$
\begin{aligned}
|\tilde{w}-w| & \leq \delta w, \\
\left|\tilde{b}_{i p}-b_{i p}\right| & \leq \delta\left(w_{i}+\left|b_{i p}\right|\right) \quad \text { for } i \in\{1: k, q\}, \quad \text { and } \\
\left|\tilde{b}_{i j}-b_{i j}\right| & \leq \delta\left|b_{i j}\right| \quad \text { for } i \neq j, i \in\{1: k, p, q\}, j \in\{1: k, q\},
\end{aligned}
$$

where $\delta=\frac{2 \epsilon}{1-\epsilon}$.

Proof. Using that $A$ is row diagonally dominant and has nonnegative diagonal entries, we have for $i \in\{1: k, q\}$,

$$
\begin{aligned}
\sum_{j \in\{1: k, p, q\} \backslash\{i\}}\left|b_{i j}\right| & =\sum_{j \in\{1: k, q\} \backslash\{i\}}\left|b_{i j}\right|+\left|b_{i p}\right|=\sum_{j \in\{1: k, q\} \backslash\{i\}}\left|a_{i j}\right|+\left|a_{i p}-\sum_{j \notin\{1: k, p, q\}} s_{j} a_{i j}\right| \\
& \leq \sum_{j \in\{1: k, q\} \backslash\{i\}}\left|a_{i j}\right|+\left|a_{i p}\right|+\sum_{j \notin\{1: k, p, q\}}\left|a_{i j}\right|=\sum_{j \neq i}\left|a_{i j}\right| \leq a_{i i}=b_{i i},
\end{aligned}
$$

and, for $i=p$,

$$
\begin{aligned}
\sum_{j \in\{1: k, q\}}\left|b_{p j}\right| & =\sum_{j \in\{1: k, q\}}\left|a_{p j}\right|=\sum_{j \neq p}\left|a_{p j}\right|-\sum_{j \notin\{1: k, p, q\}}\left|a_{p j}\right| \\
& \leq \sum_{j \neq p}\left|a_{p j}\right|-\sum_{j \notin\{1: k, p, q\}} s_{j} a_{p j} \leq a_{p p}-\sum_{j \notin\{1: k, p, q\}} s_{j} a_{p j}=b_{p p} .
\end{aligned}
$$

Hence, $B$ is row diagonally dominant with nonnegative diagonals. Using the same argument, the row diagonal dominance of $\tilde{A}$, and $\tilde{a}_{i i} \geq 0$, we can show that $\tilde{B}$ is row diagonally dominant with nonnegative diagonals as well. Thus, we can parameterize $B$ and $\tilde{B}$ in terms of their diagonally dominant parts and off-diagonal entries. Let $B=\mathcal{D}\left(B_{D}, w\right)$ and $\tilde{B}=\mathcal{D}\left(\tilde{B}_{D}, \tilde{w}\right)$ with $w=\left[w_{i}\right]$ and $\tilde{w}=\left[\tilde{w}_{i}\right] \in \mathbb{R}^{k+2}$. Now, note that, for $i \in\{1: k, p, q\}, j \in\{1: k, q\}$, and $i \neq j$, we have

$$
\left|\tilde{b}_{i j}-b_{i j}\right|=\left|\tilde{a}_{i j}-a_{i j}\right| \leq \epsilon\left|a_{i j}\right|=\epsilon\left|b_{i j}\right|,
$$

and, for $i \in\{1: k, q\}$ and $j=p$, we use $\left|a_{i p}\right| \leq\left|a_{i p}\right|+v_{i}$ to get

$$
\begin{aligned}
\left|\tilde{b}_{i p}-b_{i p}\right| & =\left|\left(\tilde{a}_{i p}-\sum_{j \notin\{1: k, p, q\}} s_{j} \tilde{a}_{i j}\right)-\left(a_{i p}-\sum_{j \notin\{1: k, p, q\}} s_{j} a_{i j}\right)\right| \\
& \leq\left|\tilde{a}_{i p}-a_{i p}\right|+\sum_{j \notin\{1: k, p, q\}}\left|\tilde{a}_{i j}-a_{i j}\right| \leq \epsilon\left(\left|a_{i p}\right|+v_{i}\right)+\epsilon \sum_{j \notin\{1: k, p, q\}}\left|a_{i j}\right| \\
& =\epsilon\left(\sum_{j \notin\{1: k, q\}}\left|a_{i j}\right|+v_{i}\right)=\epsilon\left(\sum_{j \notin\{1: k, q\}}\left|a_{i j}\right|+a_{i i}-\sum_{j \neq i}\left|a_{i j}\right|\right) \\
& =\epsilon\left(a_{i i}-\sum_{j \in\{1: k, q\} \backslash\{i\}}\left|a_{i j}\right|\right)=\epsilon\left(b_{i i}-\sum_{j \in\{1: k, q\} \backslash\{i\}}\left|b_{i j}\right|\right) \\
& =\epsilon\left(b_{i i}-\sum_{j \in\{1: k, p, q\} \backslash\{i\}}\left|b_{i j}\right|+\left|b_{i p}\right|\right)=\epsilon\left(w_{i}+\left|b_{i p}\right|\right) .
\end{aligned}
$$

Copyright $(\odot$ by SIAM. Unauthorized reproduction of this article is prohibited. 
Thus, we have proved (3.30)-(3.31) for the off-diagonal entries of $B$ and $\tilde{B}$. Now we focus on the diagonally dominant parts. Let $i \in\{1: k, q\}$ and observe

$$
\begin{aligned}
w_{i} & =b_{i i}-\sum_{j \in\{1: k, p, q\} \backslash\{i\}}\left|b_{i j}\right|=b_{i i}-\sum_{j \in\{1: k, q\} \backslash\{i\}}\left|b_{i j}\right|-\left|b_{i p}\right| \\
& =a_{i i}-\sum_{j \in\{1: k, q\} \backslash\{i\}}\left|a_{i j}\right|-\left|a_{i p}-\sum_{j \notin\{1: k, p, q\}} s_{j} a_{i j}\right| \\
& =v_{i}+\sum_{j \notin\{1: k, q\}}\left|a_{i j}\right|-\left|a_{i p}-\sum_{j \notin\{1: k, p, q\}} s_{j} a_{i j}\right| \\
& =v_{i}+\sum_{j \notin\{1: k, p, q\}}\left|a_{i j}\right|+\left|a_{i p}\right|-\left|a_{i p}-\sum_{j \notin\{1: k, p, q\}} s_{j} a_{i j}\right| .
\end{aligned}
$$

Similarly, we have

$$
\tilde{w}_{i}=\tilde{v}_{i}+\sum_{j \notin\{1: k, p, q\}}\left|\tilde{a}_{i j}\right|+\left|\tilde{a}_{i p}\right|-\left|\tilde{a}_{i p}-\sum_{j \notin\{1: k, p, q\}} s_{j} \tilde{a}_{i j}\right| .
$$

Next, we will consider two cases.

Case 1: $\operatorname{sign}\left(a_{i p}-\sum_{j \notin\{1: k, p, q\}} s_{j} a_{i j}\right)=\operatorname{sign}\left(\tilde{a}_{i p}-\sum_{j \notin\{1: k, p, q\}} s_{j} \tilde{a}_{i j}\right)=: \theta$. Then,

$$
\begin{aligned}
\tilde{w}_{i} & =\tilde{v}_{i}+\sum_{j \notin\{1: k, p, q\}}\left|\tilde{a}_{i j}\right|+\left|\tilde{a}_{i p}\right|-\theta\left(\tilde{a}_{i p}-\sum_{j \notin\{1: k, p, q\}} s_{j} \tilde{a}_{i j}\right) \\
& =\tilde{v}_{i}+\sum_{j \notin\{1: k, p, q\}}\left|\tilde{a}_{i j}\right|+\left|\tilde{a}_{i p}\right|-\theta \tilde{a}_{i p}+\theta \sum_{j \notin\{1: k, p, q\}} s_{j} \tilde{a}_{i j} \\
& =\tilde{v}_{i}+\sum_{j \notin\{1: k, p, q\}}\left|\tilde{a}_{i j}\right|\left(1+\theta s_{j} \operatorname{sign}\left(\tilde{a}_{i j}\right)\right)+\left|\tilde{a}_{i p}\right|\left(1-\theta \operatorname{sign}\left(\tilde{a}_{i p}\right)\right) \\
& =\tilde{v}_{i}+\sum_{j \notin\{1: k, p, q\}}\left|\tilde{a}_{i j}\right|\left(1+\theta s_{j} \operatorname{sign}\left(a_{i j}\right)\right)+\left|\tilde{a}_{i p}\right|\left(1-\theta \operatorname{sign}\left(a_{i p}\right)\right),
\end{aligned}
$$

where we have used that $\operatorname{sign}\left(\tilde{a}_{i j}\right)=\operatorname{sign}\left(a_{i j}\right)$ for all $j \neq i$. Similarly, we have

$$
w_{i}=v_{i}+\sum_{j \notin\{1: k, p, q\}}\left|a_{i j}\right|\left(1+\theta s_{j} \operatorname{sign}\left(a_{i j}\right)\right)+\left|a_{i p}\right|\left(1-\theta \operatorname{sign}\left(a_{i p}\right)\right)
$$

and, hence,

$$
\begin{aligned}
\left|\tilde{w}_{i}-w_{i}\right| \leq & \left|\tilde{v}_{i}-v_{i}\right|+\sum_{j \notin\{1: k, p, q\}}\left|\tilde{a}_{i j}-a_{i j}\right|\left(1+\theta s_{j} \operatorname{sign}\left(a_{i j}\right)\right) \\
& +\left|\tilde{a}_{i p}-a_{i p}\right|\left(1-\theta \operatorname{sign}\left(a_{i p}\right)\right) \\
\leq & \epsilon v_{i}+\epsilon \sum_{j \notin\{1: k, p, q\}}\left|a_{i j}\right|\left(1+\theta s_{j} \operatorname{sign}\left(a_{i j}\right)\right)+\epsilon\left|a_{i p}\right|\left(1-\theta \operatorname{sign}\left(a_{i p}\right)\right) \\
\leq & \epsilon w_{i} .
\end{aligned}
$$

Copyright (? by SIAM. Unauthorized reproduction of this article is prohibited. 
Case 2: $\operatorname{sign}\left(a_{i p}-\sum_{j \notin\{1: k, p, q\}} s_{j} a_{i j}\right) \neq \operatorname{sign}\left(\tilde{a}_{i p}-\sum_{j \notin\{1: k, p, q\}} s_{j} \tilde{a}_{i j}\right)$. In this case,

$$
\begin{aligned}
\left|\tilde{a}_{i p}-\sum_{j \notin\{1: k, p, q\}} s_{j} \tilde{a}_{i j}\right| & +\left|a_{i p}-\sum_{j \notin\{1: k, p, q\}} s_{j} a_{i j}\right| \\
& =\left|\left(\tilde{a}_{i p}-\sum_{j \notin\{1: k, p, q\}} s_{j} \tilde{a}_{i j}\right)-\left(a_{i p}-\sum_{j \notin\{1: k, p, q\}} s_{j} a_{i j}\right)\right| \\
& \leq\left|\tilde{a}_{i p}-a_{i p}\right|+\sum_{j \notin\{1: k, p, q\}}\left|\tilde{a}_{i j}-a_{i j}\right| \\
& \leq \epsilon\left|a_{i p}\right|+\epsilon \sum_{j \notin\{1: k, p, q\}}\left|a_{i j}\right|,
\end{aligned}
$$

which, combined with (3.32) and (3.33), yields

$$
\begin{aligned}
\left|\tilde{w}_{i}-w_{i}\right|= & \mid\left(\tilde{v}_{i}+\sum_{j \notin\{1: k, q\}}\left|\tilde{a}_{i j}\right|-\left|\tilde{a}_{i p}-\sum_{j \notin\{1: k, p, q\}} s_{j} \tilde{a}_{i j}\right|\right) \\
& -\left(v_{i}+\sum_{j \notin\{1: k, q\}}\left|a_{i j}\right|-\left|a_{i p}-\sum_{j \notin\{1: k, p, q\}} s_{j} a_{i j}\right|\right) \mid \\
\leq & \left|\tilde{v}_{i}-v_{i}\right|+\sum_{j \notin\{1: k, q\}}|| \tilde{a}_{i j}|-| a_{i j}|| \\
& +\left|\tilde{a}_{i p}-\sum_{j \notin\{1: k, p, q\}} s_{j} \tilde{a}_{i j}\right|+\left|a_{i p}-\sum_{j \notin\{1: k, p, q\}} s_{j} a_{i j}\right| \\
\leq & \epsilon v_{i}+\epsilon \sum_{j \notin\{1: k, q\}}\left|a_{i j}\right|+\epsilon \sum_{j \notin\{1: k, q\}}\left|a_{i j}\right| \\
\leq & 2 \epsilon\left(\sum_{j \notin\{1: k, q\}}\left|a_{i j}\right|\right) .
\end{aligned}
$$

So, from (3.32) and (3.35),

$$
\begin{aligned}
w_{i} & =v_{i}+\sum_{j \notin\{1: k, q\}}\left|a_{i j}\right|-\left|a_{i p}-\sum_{j \notin\{1: k, p, q\}} s_{j} a_{i j}\right| \geq v_{i}+\sum_{j \notin\{1: k, q\}}\left|a_{i j}\right|-\epsilon \sum_{j \notin\{1: k, q\}}\left|a_{i j}\right| \\
& \geq(1-\epsilon)\left(v_{i}+\sum_{j \notin\{1: k, q\}}\left|a_{i j}\right|\right) .
\end{aligned}
$$

Combining this inequality and (3.36), we have

$$
\left|\tilde{w}_{i}-w_{i}\right| \leq \frac{2 \epsilon}{1-\epsilon} w_{i}
$$

The inequalities (3.34) and (3.37) prove the bound (3.29) for $w_{i}$ with $i \in\{1: k, q\}$.

Copyright (C) by SIAM. Unauthorized reproduction of this article is prohibited. 
Finally, we prove (3.29) for $i=p$. Note that

$$
\begin{aligned}
w_{p} & =b_{p p}-\sum_{j \in\{1: k, q\}}\left|b_{p j}\right|=a_{p p}-\sum_{j \notin\{1: k, p, q\}} s_{j} a_{p j}-\sum_{j \in\{1: k, q\}}\left|a_{p j}\right| \\
& =v_{p}+\sum_{j \neq p}\left|a_{p j}\right|-\sum_{j \notin\{1: k, p, q\}} s_{j} a_{p j}-\sum_{j \in\{1: k, q\}}\left|a_{p j}\right| \\
& =v_{p}+\sum_{j \notin\{1: k, p, q\}}\left|a_{p j}\right|-\sum_{j \notin\{1: k, p, q\}} s_{j} a_{p j}=v_{p}+\sum_{j \notin\{1: k, p, q\}}\left(\left|a_{p j}\right|-s_{j} a_{p j}\right) \\
& =v_{p}+\sum_{j \notin\{1: k, p, q\}}\left|a_{p j}\right|\left(1-s_{j} \operatorname{sign}\left(a_{p j}\right)\right) .
\end{aligned}
$$

Similarly, we have

$$
\tilde{w}_{p}=\tilde{v}_{p}+\sum_{j \notin\{1: k, p, q\}}\left|\tilde{a}_{p j}\right|\left(1-s_{j} \operatorname{sign}\left(\tilde{a}_{p j}\right)\right)=\tilde{v}_{p}+\sum_{j \notin\{1: k, p, q\}}\left|\tilde{a}_{p j}\right|\left(1-s_{j} \operatorname{sign}\left(a_{p j}\right)\right),
$$

$\operatorname{since} \operatorname{sign}\left(\tilde{a}_{p j}\right)=\operatorname{sign}\left(a_{p j}\right)$. Thus,

$$
\begin{aligned}
\left|\tilde{w}_{p}-w_{p}\right| & \leq\left|\tilde{v}_{p}-v_{p}\right|+\sum_{j \notin\{1: k, p, q\}}\left|\tilde{a}_{p j}-a_{p j}\right|\left(1-s_{j} \operatorname{sign}\left(a_{p j}\right)\right) \\
& \leq \epsilon v_{p}+\epsilon \sum_{j \notin\{1: k, p, q\}}\left|a_{p j}\right|\left(1-s_{j} \operatorname{sign}\left(a_{p j}\right)\right)=\epsilon w_{p} .
\end{aligned}
$$

So, we have that $\left|\tilde{w}_{i}-w_{i}\right| \leq \frac{2 \epsilon}{1-\epsilon} w_{i}$ for all $i \in\{1: k, p, q\}$. Lemma 3.9 is proved.

The next lemma relates one of the minors of the matrix $B$ defined in Lemma 3.9 with one minor of $A$. In the statement, we use the notation introduced in (3.4).

Lemma 3.10. Let $A$ and $B$ be defined as in Lemma 3.9 and define

$$
\left(g_{B}\right)_{p p}^{(k+1)}:=\operatorname{det} B([1: k, p],[1: k, p]) .
$$

Then, we have

(a) if $g_{k k}^{(k)} \neq 0$, let $A^{(k+1)}=\left[a_{i j}^{(k+1)}\right]$ be the row diagonally dominant matrix with nonnegative diagonal entries obtained after $k$ stages of Gaussian elimination have been performed on $A$, and let $A^{(k+1)}$ be parameterized as $A^{(k+1)}=$ $\mathcal{D}\left(A_{D}^{(k+1)}, v^{(k+1)}\right)$, with $v^{(k+1)}=\left[v_{i}^{(k+1)}\right]$. Then

$$
\left(g_{B}\right)_{p p}^{(k+1)}=\left(v_{p}^{(k+1)}+\left|a_{p q}^{(k+1)}\right|\right) g_{k k}^{(k)}
$$

(b) if $g_{k k}^{(k)}=0$, then $\left(g_{B}\right)_{p p}^{(k+1)}=0$.

Proof. Observe that $B([1: k, p],[1: k, p])$ and $A([1: k, p],[1: k, p])$ have columns 1 through $k$ equal and for the last column, we have

$$
B([1: k, p], p)=A([1: k, p], p)-\sum_{j \notin\{1: k, p, q\}} s_{j} A([1: k, p], j) .
$$

Using the fact that the determinant is a linear function of any of its columns, assuming that the remaining columns are fixed, we obtain

$$
\left(g_{B}\right)_{p p}^{(k+1)}=\operatorname{det} A([1: k, p],[1: k, p])-\sum_{j \notin\{1: k, p, q\}} s_{j} \operatorname{det} A([1: k, p],[1: k, j]) .
$$

Copyright (? by SIAM. Unauthorized reproduction of this article is prohibited. 
If $g_{k k}^{(k)}=\operatorname{det} A(1: k, 1: k) \neq 0$, then

$\left(g_{B}\right)_{p p}^{(k+1)}=g_{k k}^{(k)}\left(\frac{\operatorname{det} A([1: k, p],[1: k, p])}{\operatorname{det} A(1: k, 1: k)}-\sum_{j \notin\{1: k, p, q\}} s_{j} \frac{\operatorname{det} A([1: k, p],[1: k, j])}{\operatorname{det} A(1: k, 1: k)}\right)$.

By (2.5),

$\left(g_{B}\right)_{p p}^{(k+1)}=g_{k k}^{(k)}\left(a_{p p}^{(k+1)}-\sum_{j \notin\{1: k, p, q\}} s_{j} a_{p j}^{(k+1)}\right)=g_{k k}^{(k)}\left(a_{p p}^{(k+1)}-\sum_{j \notin\{1: k, p, q\}}\left|a_{p j}^{(k+1)}\right|\right)$.

Since, $a_{p j}^{(k+1)}=0$ for $1 \leq j \leq k$, we get

$$
\left(g_{B}\right)_{p p}^{(k+1)}=g_{k k}^{(k)}\left(a_{p p}^{(k+1)}-\sum_{j \neq p}\left|a_{p j}^{(k+1)}\right|+\left|a_{p q}^{(k+1)}\right|\right)=g_{k k}^{(k)}\left(v_{p}^{(k+1)}+\left|a_{p q}^{(k+1)}\right|\right),
$$

which proves part (a). Next, we prove part (b). If $g_{k k}^{(k)}=\operatorname{det} A(1: k, 1: k)=0$, then one of the pivots $a_{j j}^{(j)}$, for $1 \leq j \leq k$, in the Gaussian elimination for $A$ must be 0 . Since $A^{(j)}$ is still row diagonally dominant, then the $j$ th row of $A^{(j)}$ must be entirely 0 . Then applying $j-1$ stages of Gaussian elimination to the row diagonally dominant matrix $B([1: k, p],[1: k, p])$ produces also a zero $j$ th pivot and the $j$ th row is also entirely 0 . Hence $\left(g_{B}\right)_{p p}^{(k+1)}=\operatorname{det} B([1: k, p],[1: k, p])=0$.

We now present Lemma 3.11 that establishes a different bound than the one in [14, Lemma 7(b)] for the perturbation of the nonprincipal minors defined in (3.4) under the standard perturbations defined in (3.2). This lemma is a consequence of the considerable effort we have made so far on studying structured perturbations of minors of row diagonally dominant matrices. It will allow us to prove Theorem 3.2.

Lemma 3.11. Let $A=\mathcal{D}\left(A_{D}, v\right) \in \mathbb{R}^{n \times n}$ be such that $v \geq 0$ and let $\tilde{A}=$ $\mathcal{D}\left(\tilde{A}_{D}, \tilde{v}\right) \in \mathbb{R}^{n \times n}$ be a matrix that satisfies

$$
|\tilde{v}-v| \leq \epsilon v \quad \text { and } \quad\left|\tilde{A}_{D}-A_{D}\right| \leq \epsilon\left|A_{D}\right| \quad \text { for some } 0 \leq \epsilon<\frac{1}{5} .
$$

Let $1 \leq k \leq n-2, k+1 \leq p, q \leq n$, and $p \neq q$. Then, we have

(a) if $g_{k k}^{(k)} \neq 0$, let $A^{(k+1)}=\left[a_{i j}^{(k+1)}\right]=\mathcal{D}\left(A_{D}^{(k+1)}, v^{(k+1)}\right)$, with $v^{(k+1)}=\left[v_{i}^{(k+1)}\right]$, be the row diagonally dominant matrix with nonnegative diagonal entries obtained after $k$ stages of Gaussian elimination have been performed on A. Then

$$
\left|\tilde{g}_{p q}^{(k+1)}-g_{p q}^{(k+1)}\right| \leq \frac{4}{3}\left(\left(1+\epsilon_{0}\right)^{k+1}-1\right)\left(v_{p}^{(k+1)}+\left|a_{p q}^{(k+1)}\right|\right) g_{k k}^{(k)},
$$

where $\epsilon_{0}=\frac{6 \epsilon}{1-\epsilon}$;

(b) if $g_{k k}^{(k)}=0$, then $\tilde{g}_{p q}^{(k+1)}=g_{p q}^{(k+1)}=0$.

Proof. Suppose $g_{k k}^{(k)} \neq 0$. Define $B$ and $\tilde{B}$ as in Lemma 3.9. By (3.29)-(3.31), we can apply Lemma 3.8 to the minors of $B$ and $\tilde{B}$ defined in (3.23) to obtain

$$
\left|\left(\tilde{g}_{B}\right)_{p q}^{(k+1)}-\left(g_{B}\right)_{p q}^{(k+1)}\right| \leq \frac{4}{3}\left((1+3 \delta)^{k+1}-1\right)\left(g_{B}\right)_{p p}^{(k+1)},
$$

Copyright $@$ by SIAM. Unauthorized reproduction of this article is prohibited. 
with $\delta=2 \epsilon /(1-\epsilon)$. By the construction of $B$ and $\tilde{B}$, we have

$$
\left(\tilde{g}_{B}\right)_{p q}^{(k+1)}=\tilde{g}_{p q}^{(k+1)} \text { and }\left(g_{B}\right)_{p q}^{(k+1)}=g_{p q}^{(k+1)},
$$

and hence, from (3.39),

$$
\left|\tilde{g}_{p q}^{(k+1)}-g_{p q}^{(k+1)}\right| \leq \frac{4}{3}\left((1+3 \delta)^{k+1}-1\right)\left(g_{B}\right)_{p p}^{(k+1)} .
$$

Next, apply Lemma 3.10(a) to get

$$
\left|\tilde{g}_{p q}^{(k+1)}-g_{p q}^{(k+1)}\right| \leq \frac{4}{3}\left((1+3 \delta)^{k+1}-1\right)\left(v_{p}^{(k+1)}+\left|a_{p q}^{(k+1)}\right|\right) g_{k k}^{(k)},
$$

which proves part (a).

Next we prove part (b). We have seen in the proof of Lemma 3.10 that $g_{k k}^{(k)}=0$ implies that one of the pivots $a_{j j}^{(j)}$ (for $1 \leq j \leq k$ ) in the Gaussian elimination for $A$ must be 0 . Since $A^{(j)}$ is still row diagonally dominant, the $j$ th row of $A^{(j)}$ must be entirely 0 . Then applying $j-1$ stages of the Gaussian elimination to $A([1: k, p],[1: k, q])$ produces a $j$ th row which is entirely 0 and hence $g_{p q}^{(k+1)}=0$. Furthermore, by (3.20) in Lemma 3.7, we have that $g_{k k}^{(k)}=0$ implies $\tilde{g}_{k k}^{(k)}=0$ and the same argument we have used above for $A$ can be used on $\tilde{A}$ to prove $\tilde{g}_{p q}^{(k+1)}=0$.

The results presented so far in section 3.1 are valid for general row diagonally dominant matrices with nonnegative diagonal. From now on, we assume that the matrix $A$ is arranged for column diagonal dominance pivoting. This allows us to bound the sum of the absolute values of the entries below the diagonal of each column of the $L$ factor in terms of the diagonally dominant parts of the corresponding Schur complement and the corresponding pivot, as seen in Lemma 3.12.

Lemma 3.12. Let $A=\mathcal{D}\left(A_{D}, v\right) \in \mathbb{R}^{n \times n}$ be such that $v \geq 0$ and assume that $A$ is arranged for the column diagonal dominance pivoting strategy. Let $A^{(k)}=$ $\mathcal{D}\left(A_{D}^{(k)}, v^{(k)}\right)$, with $A^{(k)}=\left[a_{i j}^{(k)}\right]$ and $v^{(k)}=\left[v_{i}^{(k)}\right]$, be the row diagonally dominant matrix with nonnegative diagonal entries obtained after $k-1$ stages of Gaussian elimination have been applied on $A$. Then, for $k \leq \operatorname{rank}(A)$, we have

$$
\sum_{i=k+1}^{n}\left(\left|a_{i k}^{(k)}\right|+v_{i}^{(k)}\right) \leq(n-k) a_{k k}^{(k)}
$$

Proof. According to (2.6), define $\delta_{i}^{(k)}:=a_{i i}^{(k)}-\sum_{j=k, j \neq i}^{n}\left|a_{j i}^{(k)}\right|$. Then, we have

$$
a_{k k}^{(k)}=\max _{k \leq i \leq n}\left\{a_{i i}^{(k)}: \delta_{i}^{(k)} \geq 0\right\} .
$$

If $\delta_{i}^{(k)} \geq 0$ for all $i=k+1, \ldots, n$, then

$$
\sum_{i=k+1}^{n}\left(\left|a_{i k}^{(k)}\right|+v_{i}^{(k)}\right) \leq \sum_{i=k+1}^{n} a_{i i}^{(k)} \leq \sum_{i=k+1}^{n} a_{k k}^{(k)} \leq(n-k) a_{k k}^{(k)},
$$

which proves the result in this case. Otherwise, if there is at least one $\delta_{i}^{(k)}<0$ for some $i=k+1, \ldots, n$, then from the definition of $v_{i}^{(k)}$ we obtain, for $k+1 \leq i \leq n$,

$$
\left|a_{i k}^{(k)}\right|+v_{i}^{(k)}=a_{i i}^{(k)}-\sum_{j=k+1, j \neq i}^{n}\left|a_{i j}^{(k)}\right|
$$

Copyright (? by SIAM. Unauthorized reproduction of this article is prohibited. 
and sum over $i$ to obtain

$$
\begin{aligned}
& \sum_{i=k+1}^{n}\left(\left|a_{i k}^{(k)}\right|+v_{i}^{(k)}\right) \\
& =\sum_{i=k+1}^{n} a_{i i}^{(k)}-\sum_{i=k+1}^{n} \sum_{j=k+1, j \neq i}^{n}\left|a_{i j}^{(k)}\right|=\sum_{i=k+1}^{n} a_{i i}^{(k)}-\sum_{i=k+1}^{n} \sum_{j=k+1, j \neq i}^{n}\left|a_{j i}^{(k)}\right| \\
& =\sum_{i=k+1}^{n}\left(a_{i i}^{(k)}-\sum_{j=k+1, j \neq i}^{n}\left|a_{j i}^{(k)}\right|\right)=\sum_{i=k+1}^{n}\left(a_{i i}^{(k)}-\sum_{j=k, j \neq i}^{n}\left|a_{j i}^{(k)}\right|+\left|a_{k i}^{(k)}\right|\right) \\
& =\sum_{i=k+1}^{n}\left(\delta_{i}^{(k)}+\left|a_{k i}^{(k)}\right|\right) \leq a_{k k}^{(k)}+\sum_{i=k+1}^{n} \delta_{i}^{(k)} \\
& \leq a_{k k}^{(k)}+\sum_{i=k+1, \delta_{i}^{(k)} \geq 0}^{n} \delta_{i}^{(k)} \leq a_{k k}^{(k)}+\sum_{i=k+1, \delta_{i}^{(k)} \geq 0}^{n} a_{i i}^{(k)} \\
& \leq a_{k k}^{(k)}+\sum_{i=k+1, \delta_{i}^{(k)} \geq 0}^{n} a_{k k}^{(k)} \leq a_{k k}^{(k)}+(n-k-1) a_{k k}^{(k)} \\
& =(n-k) a_{k k}^{(k)},
\end{aligned}
$$

since for $\delta_{i}^{(k)} \geq 0, \delta_{i}^{(k)} \leq a_{i i}^{(k)} \leq a_{k k}^{(k)}$.

Finally, we are now ready to present the proof of Theorem 3.2.

Proof of Theorem 3.2. As a consequence of Theorem 3.1(a), we have $\operatorname{rank}(A)=$ $\operatorname{rank}(\tilde{A})$. Therefore, from Definition 2.4, it is observed that we only need to pay attention to the variation of the strictly lower triangular entries of $L$ in its first $r:=$ $\operatorname{rank}(A)$ columns. Using (2.2), Lemma 3.11(a) with $p=i, q=j$, and $k=j-1$, and [14, Lemma 4(b)], we have, for $i>j$ and $1 \leq j \leq r$,

$$
\tilde{l}_{i j}=\frac{\tilde{g}_{i j}^{(j)}}{\tilde{g}_{j j}^{(j)}}=\frac{g_{i j}^{(j)}+\frac{4}{3} \chi\left(v_{i}^{(j)}+\left|a_{i j}^{(j)}\right|\right) g_{j-1, j-1}^{(j-1)}}{g_{j j}^{(j)}\left(1+\xi_{1}\right) \cdots\left(1+\xi_{j}\right)},
$$

where $\left|\xi_{1}\right| \leq \epsilon, \ldots,\left|\xi_{j}\right| \leq \epsilon$ and $|\chi| \leq\left(\left(1+\epsilon_{0}\right)^{j}-1\right)$. Define

$$
\zeta:=\frac{1}{\left(1+\xi_{1}\right) \cdots\left(1+\xi_{j}\right)}-1
$$

and note $|\zeta| \leq \frac{1}{(1-\epsilon)^{j}}-1$. Hence, from (3.41) and (2.5),

$\tilde{l}_{i j}=\left(l_{i j}+\frac{\frac{4}{3} \chi\left(v_{i}^{(j)}+\left|a_{i j}^{(j)}\right|\right)}{a_{j j}^{(j)}}\right)(1+\zeta)$ and $\tilde{l}_{i j}-l_{i j}=\zeta l_{i j}+\frac{\frac{4}{3} \chi(1+\zeta)\left(v_{i}^{(j)}+\left|a_{i j}^{(j)}\right|\right)}{a_{j j}^{(j)}}$.

Taking the absolute value gives

$$
\left|\tilde{l}_{i j}-l_{i j}\right| \leq|\zeta|\left|l_{i j}\right|+\frac{4}{3}|\chi||1+\zeta| \frac{v_{i}^{(j)}+\left|a_{i j}^{(j)}\right|}{a_{j j}^{(j)}}
$$

and then summing over $i$ yields

$$
\sum_{i=j+1}^{n}\left|\tilde{l}_{i j}-l_{i j}\right| \leq|\zeta| \sum_{i=j+1}^{n}\left|l_{i j}\right|+\frac{4}{3}|\chi||1+\zeta| \frac{\sum_{i=j+1}^{n}\left(v_{i}^{(j)}+\left|a_{i j}^{(j)}\right|\right)}{a_{j j}^{(j)}} .
$$

Copyright (? by SIAM. Unauthorized reproduction of this article is prohibited. 
By assumption $A$ is arranged for column diagonal dominance pivoting, which means that the matrix $L$ is column diagonally dominant, that is, $\sum_{i=j+1}^{n}\left|l_{i j}\right| \leq 1$ for all $j$. Use this fact and Lemma 3.12 in (3.42) to get, for $1 \leq j \leq r$,

$$
\begin{aligned}
\sum_{i=j+1}^{n}\left|\tilde{l}_{i j}-l_{i j}\right| & \leq|\zeta|+\frac{4}{3}|\chi||1+\zeta| \frac{(n-1) a_{j j}^{(j)}}{a_{j j}^{(j)}}=|\zeta|+\frac{4}{3}(n-1)|\chi||1+\zeta| \\
& \leq\left(\frac{1}{(1-\epsilon)^{n}}-1\right)+\frac{4}{3}(n-1)\left(\left(1+\epsilon_{0}\right)^{n}-1\right) \frac{1}{(1-\epsilon)^{n}} \\
& \leq \frac{n(4 n-1) \epsilon_{0}}{3\left(1-2 n \epsilon_{0}\right)}=\frac{2 n(4 n-1) \epsilon}{1-(12 n+1) \epsilon},
\end{aligned}
$$

where we have used $\epsilon<\epsilon_{0}=6 \epsilon /(1-\epsilon)$, standard results from [24, Chap. 3], and $(12 n+1) \epsilon<1$. Now, Theorem 3.2 follows from observing that

$$
\|\tilde{L}-L\|_{1}=\max _{1 \leq j \leq(n-1)} \sum_{i=j+1}^{n}\left|\tilde{l}_{i j}-l_{i j}\right| .
$$

4. Conclusions and future work. We have proved that small componentwise relative perturbations in the diagonally dominant parts and off-diagonal entries of row diagonally dominant matrices with nonnegative diagonal entries produce small relative normwise perturbations in the $L$ factor obtained by applying the column diagonal dominance pivoting strategy to this type of matrix. This result combined with the perturbation results for the $D$ and $U$ factors presented in [14] show that the column diagonal dominance pivoting strategy for row diagonally dominant matrices leads, simultaneously, to LDU factorizations that are guaranteed to be rank-revealing decompositions, i.e., the factors $L$ and $U$ are guaranteed to have small condition numbers, and that always undergo small relative perturbations under the structured perturbations considered in this work. The perturbation results presented in this paper are fundamental to prove in [10] that essentially all interesting magnitudes corresponding to row diagonally dominant matrices undergo small relative variations under small relative perturbations in the diagonally dominant parts and off-diagonal entries and, therefore, that these magnitudes can be computed with high accuracy by algorithms based on rank-revealing decompositions [5, 11, 15, 17, 39].

Acknowledgment. The authors thank two anonymous referees for many suggestions that have helped us to improve this manuscript.

\section{REFERENCES}

[1] A. S. Alfa, J. Xue, And Q. Ye, Entrywise perturbation theory for diagonally dominant Mmatrices with applications, Numer. Math., 90 (2002), pp. 401-414.

[2] A. Barrlund, Perturbation bounds for the $L D L^{H}$ and $L U$ decompositions, BIT, 31 (1991), pp. 358-363.

[3] S. BORA, Structured eigenvalue condition number and backward error of a class of polynomial eigenvalue problems, SIAM J. Matrix Anal. Appl., 31 (2010), pp. 900-917.

[4] S. Bora And V. Mehrmann, Linear perturbation theory for structured matrix pencils arising in control theory, SIAM J. Matrix Anal. Appl., 28 (2006), pp. 148-169.

[5] N. Castro-González, J. Ceballos, F. M. Dopico, and J. M. Molera, Accurate solution of structured least squares problems via rank-revealing decompositions, SIAM J. Matrix Anal. Appl., 34 (2013), pp. 1112-1128.

[6] X.-W. Chang, Some features of Gaussian elimination with rook pivoting, BIT, 42 (2002), pp. $66-83$. 
[7] X.-W. Chang And R.-C. LI, Multiplicative perturbation analysis for QR factorizations, Numer. Algebra Control Optim., 1 (2011), pp. 301-316.

[8] X.-W. Chang and C. C. Paige, On the sensitivity of the LU factorization, BIT, 38 (1998), pp. $486-501$.

[9] X.-W. Chang and D. Stehlé, Rigorous perturbation bounds of some matrix factorizations, SIAM J. Matrix Anal. Appl., 31 (2010), pp. 2841-2859.

[10] M. Dailey, F. M. Dopico, AND Q. Ye, Relative perturbation theory for diagonally dominant matrices, submitted.

[11] J. Demmel, M. Gu, S. Eisenstat, I. Slapničar, K. Veselić, and Z. Drmač, Computing the singular value decomposition with high relative accuracy, Linear Algebra Appl., 299 (1999), pp. 21-80.

[12] J. Demmel and K. Veselić, Jacobi's method is more accurate than $Q R$, SIAM J. Matrix Anal. Appl., 13 (1992), pp. 1204-1245.

[13] F. M. Dopico AND P. Koev, Accurate symmetric rank revealing and eigendecompositions of symmetric structured matrices, SIAM J. Matrix Anal. Appl., 28 (2006), pp. 1126-1156.

[14] F. M. Dopico AND P. Koev, Perturbation theory for the LDU factorization and accurate computations for diagonally dominant matrices, Numer. Math., 119 (2011), pp. 337-371.

[15] F. M. Dopico, P. Koev, And J. M. Molera, Implicit standard Jacobi gives high relative accuracy, Numer. Math., 113 (2009), pp. 519-553.

[16] F. M. Dopico And J. M. Molera, Perturbation theory for factorizations of LU type through series expansions, SIAM J. Matrix Anal. Appl., 27 (2005), pp. 561-581.

[17] F. M. Dopico And J. M. Molera, Accurate solution of structured linear systems via rankrevealing decompositions, IMA J. Numer. Anal., 32 (2012), pp. 1096-1116.

[18] Z. DrmaČ And K. Veselić, New fast and accurate Jacobi SVD algorithm. I, SIAM J. Matrix Anal. Appl., 29 (2008), pp. 1322-1342.

[19] F. R. Gantmacher, The Theory of Matrices, Chelsea, New York, 1959.

[20] G. Golub and C. Van Loan, Matrix Computations, 3rd ed., Johns Hopkins University Press, Baltimore, MD, 1996.

[21] D. J. Higham and N. J. Higham, Backward error and condition of structured linear systems, SIAM J. Matrix Anal. Appl., 13 (1992), pp. 162-175.

[22] D. J. Higham And N. J. Higham, Structured backward error and condition of generalized eigenvalue problems, SIAM J. Matrix Anal. Appl., 20 (1998), pp. 493-512.

[23] N. J. Higham, A survey of componentwise perturbation theory in numerical linear algebra, in Mathematics of Computation 1943-1993: A Half Century of Computational Mathematics, AMS, Providence, RI, 1994, pp. 49-77.

[24] N. J. Higham, Accuracy and Stability of Numerical Algorithms, 2nd ed., SIAM, Philadelphia, 2002.

[25] R. A. Horn and C. R. Johnson, Topics in Matrix Analysis, Cambridge University Press, Cambridge, 1994.

[26] I. C. F. IPsen, Relative perturbation results for matrix eigenvalues and singular values, in Acta Numerica, Vol. 7, Cambridge University Press, Cambridge, 1998, pp. 151-201.

[27] M. Karow, D. Kressner, And F. Tisseur, Structured eigenvalue condition numbers, SIAM J. Matrix Anal. Appl., 28 (2006), pp. 1052-1068.

[28] R.-C. LI, Relative perturbation theory: I. Eigenvalue and singular value variations, SIAM J. Matrix Anal. Appl., 19 (1998), pp. 956-982.

[29] R.-C. LI, A bound on the solution to a structured Sylvester equation with an application to relative perturbation theory, SIAM J. Matrix Anal. Appl., 21 (2000), pp. 440-445.

[30] R.-C. Li, Y. Nakatsukasa, N. Truhar, and W.-G. Wang, Perturbation of multiple eigenvalues of Hermitian matrices, Linear Algebra Appl., 437 (2012), pp. 202-213.

[31] R.-C. Li, Y. Nakatsukasa, N. Truhar, and S. Xu, Perturbation of partitioned Hermitian definite generalized eigenvalue problems, SIAM J. Matrix Anal. Appl., 32 (2011), pp. 642663.

[32] J. M. PeñA, LDU decompositions with $L$ and $U$ well conditioned, Electron. Trans. Numer. Anal., 18 (2004), pp. 198-208.

[33] S. M. Rump, Eigenvalues, pseudospectrum and structured perturbations, Linear Algebra Appl., 413 (2006), pp. 567-593.

[34] I. SlapNIČAR, Componentwise analysis of direct factorization of real symmetric and Hermitian matrices, Linear Algebra Appl., 272 (1998), pp. 227-275.

[35] G. W. Stewart and J.-G. Sun, Matrix Perturbation Theory, Academic Press, New York, 1990.

[36] J. Sun, Componentwise perturbation bounds for some matrix decompositions, BIT, 32 (1992), pp. 702-714.

Copyright () by SIAM. Unauthorized reproduction of this article is prohibited. 
[37] F. Tisseur And N. J. Higham, Structured pseudospectra for polynomial eigenvalue problems, with applications, SIAM J. Matrix Anal. Appl., 23 (2001), pp. 187-208.

[38] K. Veselić And I. SlapniČar, Floating point perturbations of Hermitian matrices, Linear Algebra Appl., 195 (1993), pp. 81-116.

[39] Q. YE, Computing singular values of diagonally dominant matrices to high relative accuracy, Math. Comp., 77 (2008), pp. 2195-2230.

[40] Q. YE, Relative perturbation bounds for eigenvalues of symmetric positive definite diagonally dominant matrices, SIAM J. Matrix Anal. Appl., 31 (2009), pp. 11-17.

Copyright (? by SIAM. Unauthorized reproduction of this article is prohibited. 